\title{
Standard error estimation by an automated blocking method
}

\author{
Marius Jonsson \\ Department of Physics, University of Oslo, N-0316 Oslo, Norway
}

(Received 15 May 2018; revised manuscript received 16 August 2018; published 15 October 2018)

\begin{abstract}
The sample mean $\bar{X}$ is probably the most popular estimator of the expected value in all sciences and $\operatorname{var}(\bar{X})$ measures the error (standard- and mean-square-errors). Here, an alternative approach to estimation of $\operatorname{var}(\bar{X})$ for time series data is presented. The method has an accuracy similar to dependent bootstrapping, but scales in $O(n)$ time, and applies to stationary time series, including stationary Markov chains. The computational complexity is bounded by $12 n$ floating point operations, but this can be reduced to $n+O(1)$ in large computations. Convergence in relative error squared is faster than $n^{-1 / 2}$ and the method is insensitive to the probability distribution of the observations. It is proven that a small part of the correlation structure is relevant to the convergence rate of the method. From this, proof of the Blocking method [Flyvbjerg and Petersen, J. Chem. Phys. 91, 461 (1989)] follows as a corollary. The result is also used to propose a hypothesis test surveying the relevant part of the correlation structure. It yields a fully automatic method which is sufficiently robust to operate without supervision. An algorithm and sample code showing the implementation is available for PYTHON, $\mathrm{C}++$, and $\mathrm{R}$ [www.github.com/computative/block]. Method validation using autoregressive AR(1) and AR(2) processes and physics applications is included. Method self-evaluation is provided by bias and mean-square-error statistics. The method is easily adapted to multithread applications and data larger than computing cluster memory, such as ultralong time series or data streams. This way, the paper provides a stringent and modern treatment of the Blocking method using rigorous linear algebra, multivariate probability theory, real analysis, and Fisherian statistical inference.
\end{abstract}

DOI: 10.1103/PhysRevE.98.043304

\section{INTRODUCTION}

Estimation of the variance of sample means $\bar{X}=$ $(1 / n) \sum_{i=1}^{n} X_{i}$ is essential in natural sciences [1,2]. This is because $\bar{X}$ is a typical estimator of the expected value of the observations $X_{1}, X_{2}, \ldots$, if the observations are identically distributed with finite variance. The variance of the mean is the expected squared error of the estimate. Already in 1867, Chebyshev [3] explained this by showing that if the observations have expected value $\mu$, variance $\sigma^{2}$, and $\bar{X}$ has finite nonzero variance $\operatorname{var}(\bar{X})$, then for any real number $k>0$

$$
P\left(|\bar{X}-\mu|>k[\operatorname{var}(\bar{X})]^{1 / 2}\right) \leqslant \frac{1}{k^{2}} \quad(\text { Chebyshev's ineq. }) .
$$

Here, $P$ is the probability measure of the $X_{i}$ 's [4]. Chebyshev's inequality says that it is likely that the difference $|\bar{X}-\mu|$ is a small number. If the observations are independent and identically distributed, the variance of the mean is easily obtained by setting $\operatorname{var}(\bar{X})=\sigma^{2} / n$ [5], but for correlated data, the computation is more complicated [6]. Here, however, I show that if there is some integer $d>1$ such that $n=2^{d}$, and $X_{1}, \ldots, X_{n}$ are observations from a stationary time series, then the computational complexity is essentially the same as that of the sample mean, and one can use an automated scheme to compute it.

The method uses so called blocking transformations [7]. This refers to forming a new sample of data by taking the mean of every pair of subsequent observations. To be precise, blocking transformation number $i$ relates each element $B_{k}$ of a vector $\mathbf{B} \in \mathbb{R}^{n_{i}}$ to the elements $A_{k}$ of $\mathbf{A} \in \mathbb{R}^{n_{i-1}}$ by

$$
B_{k}=(1 / 2)\left(A_{2 k-1}+A_{2 k}\right) .
$$

Such transformations are applied in many areas of probability theory, and Flyvbjerg and Petersen [7] popularized a method where blocking transformations reduced the correlations of the data, and proposed a way to estimate the variance of $\bar{X}$. In this study, the mathematics is developed and an automation of the method is given. The rigor is similar to modern mathematics and an automation that is robust enough to operate without supervision is provided. The philosophy of Flyvbjerg and Petersen [7] is recycled, but the mathematics is different. The method validation has physics applications and experimental results quantifying all errors involved in applications. The method works by applying blocking transformations of the type given in Eq. (1) until correlation of observations is no longer significantly different from zero. The results show that the behavior of the method is specified by the autocovariance $\gamma(1)$. Furthermore, if $\gamma(1)$ is not significantly different from zero, the method has obtained the ideal estimate. Next, I developed an automated statistical test that stops the algorithm when there is no reason to believe that $\gamma(1)$ is different from zero. Hence, speedups of a thousand or more are possible because the complexity is $O(n)$ as compared to dependent bootstrapping or other methods of complexity $O\left(n^{2}\right)$ or $O(n \log n)$. I give the algorithm and sample code in [8].

The preliminary background (definitions and theorems) is scattered throughout in text blocks where they are required. 
First, proof of the blocking method is given and, second, an automation that is sufficiently robust to operate without supervision is derived to perform the calculations. In the discussion I compare the properties of the present blocking method with other relevant methods for computing the variance of the sample mean for correlated data.

\section{A. Key ideas}

First, the types of considered time series are defined.

Preliminaries 1. A set of random variables $\left\{X_{i}\right\}$ is said to be a time series if it is possible to think of the variables as being ordered as a function of time. The focus will be on infinite time series $X_{1}, X_{2}, \ldots$, but also the part of it that is possible to sample: that is, the first $n$ observations: $X_{1}, X_{2}, \ldots, X_{n}$. The random variables $\left\{X_{i}\right\}$ are said to be stationary or weakly stationary if (1) there exist $\mu \in \mathbb{R}$ such that $\left\langle X_{i}\right\rangle=\mu$ for all $i$ and (2) the covariances $\operatorname{cov}\left(X_{i}, X_{j}\right)$ only depend on the difference $h=|i-j|$ for all $1 \leqslant i, j \leqslant n$ [6]. A time series is strictly stationary if the cumulative distribution function (cdf) of all sets of the form $\left\{X_{i}, X_{i+1}, \ldots, X_{i+k}\right\}$ equals the cdf of the set $\left\{X_{i+j}, X_{i+j+1}, \ldots, X_{i+j+k}\right\}$ for all $i, j, k$ [6]. A strictly stationary time series with finite variance is stationary [9]. The function $\gamma(h)=\operatorname{cov}\left(X_{i}, X_{i+h}\right)$ is the autocovariance of $\left\{X_{j}\right\}_{j=1}^{\infty}$.

$\operatorname{var}(\bar{X})$ will be estimated by a quantity $\sigma_{k}^{2} / n_{k}$, which is subject to an error $e_{k}$, for $k \in\{0,1,2, \ldots\}$. These quantities will be defined soon. The index $k$ denotes how many sequential blocking transformations have been applied to the data. The first aim of the paper is to prove that if the autocovariance $\gamma(h) \rightarrow 0$ as $h \rightarrow \infty$, then $e_{k}$ can be made as small as you may wish, by applying enough blocking transformations:

Theorem. Assume that the stationary time series $X_{1}, X_{2}, \ldots$ has autocovariance $\gamma(h) \rightarrow 0$ as $h \rightarrow \infty$. Then, for every $\varepsilon>0$ there exists a natural number $K$ such that $\left|e_{k}\right|<\varepsilon$ if $K \leqslant k \leqslant d$ for the time series $X_{1}, X_{2}, \ldots, X_{2^{d}}$.

This begs the question of how to find the number $K$ that ensures that $e_{K}$ is not significantly different from zero. I present a hypothesis test that automatically determines this for you using a function $M_{j}$ that will be defined later:

Theorem. If $X_{1}, X_{2}, \ldots$ is a strictly stationary time series such that $\gamma(h) \rightarrow 0$ as $h \rightarrow \infty$ with $\lim _{n \rightarrow \infty} \operatorname{var}\left(\sum_{i=1}^{n} X_{i}\right)=$ $\infty$ and $\left\langle\left|X_{i}\right|^{2+a}\right\rangle<\infty$, for some $a>0$ then $M_{j}$ is a test statistic that is asymptotic $\chi_{d-j}^{2}$ distributed under the hypothesis $\gamma_{k}(1)=0$ for all $k \geqslant j$. The rejection region includes all values $M_{j}$ larger than $q_{d-j}(1-\alpha)$ for all $1 \leqslant j \leqslant d-1$.

Using this theorem, calculations are automated in six steps (see Fig. 2). Users primarily interested in the algorithm can jump ahead to Sec. III B to read more. For those interested in justification of the method, it is necessary to introduce measure of dependence on time series and under blocking transformations before starting work on the first theorem:

Preliminaries 2. If the time series has finite length $n$, it is possible to form an $n$-vector or $n$-tuple $\mathbf{X}$ containing the elements $\left\{X_{j}\right\}_{j=1}^{n}$. For any vector $\mathbf{X}$, define $\langle\mathbf{X}\rangle$ to be the vector with elements $\left\langle X_{i}\right\rangle$. A pair of random variables $X_{i}, X_{j}$ are uncorrelated if $\operatorname{cov}\left(X_{i}, X_{j}\right)=0$. A time series is uncorrelated if $\gamma(h)=0$ for all $h \neq 0$ and asymptotic uncorrelated if the autocovariance $\gamma(h) \rightarrow 0$ as $h \rightarrow \infty$. The matrix consisting of elements $(\Sigma)_{i j}=\gamma(|i-j|)$ is the covariance matrix of
$\mathbf{X}$, and $\widehat{\gamma}(h)$ is the sample covariance and $\widehat{\sigma}^{2}$ is the sample variance according to [6] if

$$
\widehat{\gamma}(h)=\frac{1}{n} \sum_{i=1}^{n-h}\left(X_{i}-\bar{X}\right)\left(X_{i+h}-\bar{X}\right) \quad \text { and } \quad \widehat{\sigma}^{2}=\widehat{\gamma}(0) \text {. }
$$

Subscripts are used on the variables to indicate that $\left(X_{k}\right)_{1},\left(X_{k}\right)_{2}, \ldots$ and $\mathbf{X}_{k}$ are subject to $k$ blocking transformations if they are related to $X_{1}, X_{2}, \ldots$ and $\mathbf{X}$ by $k$ repeated transformations of the type given in Eq. (1). Subscripts will also be used to denote the length of the vector $\mathbf{X}_{k}$ by the symbol $n_{k}$. Since $X_{1}, X_{2}, \ldots$ is subject to zero transformations, $\left\{\left(X_{0}\right)_{i}\right\}_{i=1}^{\infty}=\left\{X_{i}\right\}_{i=1}^{\infty}$ and $\mathbf{X}=\mathbf{X}_{0}$ and $n=$ $n_{0}$ is used to emphasize this. It will be shown in Lemma 1 that $\left\{\left(X_{k}\right)_{i}\right\}_{i=1}^{\infty}$ is indeed stationary if $\left\{X_{i}\right\}_{i=1}^{\infty}$ is, so it is possible to let the mean, autocovariance, and variance of the blocking-transformed variables be given subscripts to denote which blocking iteration they belong to: $\bar{X}_{k}, \sigma_{k}^{2}, \widehat{\sigma}_{k}^{2}, \gamma_{k}(h)$, $\widehat{\gamma_{k}}(h)$. Assuming $h=|i-j|$ and using the definition of the blocking transformation, Eq. (1), and the distributive property of the covariance, it is clear that

$$
\begin{aligned}
\gamma_{k+1}(h) & =\operatorname{cov}\left(\left(X_{k+1}\right)_{i},\left(X_{k+1}\right)_{j}\right) \\
& =\frac{1}{4} \operatorname{cov}\left(\left(X_{k}\right)_{2 i-1}+\left(X_{k}\right)_{2 i},\left(X_{k}\right)_{2 j-1}+\left(X_{k}\right)_{2 j}\right) \\
& = \begin{cases}\frac{1}{2} \gamma_{k}(2 h)+\frac{1}{2} \gamma_{k}(2 h+1) & \text { if } h=0, \\
\frac{1}{4} \gamma_{k}(2 h-1)+\frac{1}{2} \gamma_{k}(2 h)+\frac{1}{4} \gamma_{k}(2 h+1) & \text { else. }\end{cases}
\end{aligned}
$$

Finally, the variance of the sample mean can be expressed in terms of the autocovariance function by

$$
\begin{aligned}
\operatorname{var}(\bar{X})= & \operatorname{var}\left[\frac{1}{n} \sum_{i=1}^{n} X_{i}\right]=\frac{1}{n^{2}} \operatorname{cov}\left[\sum_{i=1}^{n} X_{i}, \sum_{j=1}^{n} X_{j}\right] \\
= & \frac{1}{n^{2}}[n \gamma(0)+(n-1) \gamma(1)+\cdots+\gamma(n-1) \\
& +(n-1) \gamma(-1)+(n-2) \gamma(-2) \\
& +\cdots+\gamma(1-n)] \\
= & \frac{\sigma^{2}}{n}+\frac{2}{n} \sum_{h=1}^{n-1}\left(1-\frac{h}{n}\right) \gamma(h) \text { if } \gamma(0)=\sigma^{2} .
\end{aligned}
$$

Using these definitions, the first theorem can be obtained.

\section{TIME SERIES BEHAVIOR UNDER BLOCKING TRANSFORMATIONS}

Section III states the idea of the announced algorithm. However, in order to understand why the algorithm works, preliminary results are required. The first result explains which part of the correlation structure is sufficient to survey, but before showing that, consider the following lemma, which will be frequently used. One of the main reasons why it is important, is that it justifies that $\gamma_{k}$ exists for all integers $k \geqslant 0$. It does this because it turns out that a stationary time series remains stationary after blocking transformations are applied. 
Lemma 1. Let $X_{1}, X_{2}, \ldots$ be a stationary time series and $\mathbf{X}$ be the vector of the first $n=2^{d}$ sequential observations from $X_{1}, X_{2}, \ldots$ Suppose $\mathbf{X}_{k}$ are the $n_{k}$ first observations of the time series $\left(X_{k}\right)_{1},\left(X_{k}\right)_{2}, \ldots$ Then, both $\left(X_{k}\right)_{1},\left(X_{k}\right)_{2}, \ldots$ and $\mathbf{X}_{k}$ are stationary. Moreover, if $\bar{X}_{k}$ is the sample mean of $\mathbf{X}_{k}$, then $\bar{X}=\bar{X}_{k}$ for all $0 \leqslant k \leqslant d-1$.

Proof. We first show that the time series $\left(X_{k}\right)_{1},\left(X_{k}\right)_{2}, \ldots$ is weakly stationary using induction. Since elements of $\left\{X_{i}\right\}_{i=1}^{\infty}$ are stationary, there is $\mu \in \mathbb{R}$ such that $\left\langle\left(X_{0}\right)_{i}\right\rangle=$ $\left\langle X_{i}\right\rangle=\mu$ for $i \geqslant 1$ and so the base case is trivially satisfied. For the induction step, write

$$
\left\langle\left(X_{k+1}\right)_{i}\right\rangle \stackrel{(1)}{=} \frac{1}{2}\left\langle\left(X_{k}\right)_{2 i-1}+\left(X_{k}\right)_{2 i}\right\rangle=\frac{1}{2}(\mu+\mu)=\mu .
$$

For the covariance, the elements of $\left\{X_{i}\right\}$ are stationary, and therefore $\operatorname{cov}\left(X_{i}, X_{j}\right)$ only depends on the difference $\mid i-$ $j \mid=h$, which proves the base case. Now, if the hypothesis is true for some $k$, then according to Eq. (3), it is true for $k+1$ since Eq. (3) says it only depends on the difference $h=$ $|i-j|$. This proves that the elements $\left\{\left(X_{k}\right)_{i}\right\}_{i=1}^{\infty}$ are stationary for all $k \geqslant 0$. The proof works for any smaller time series $\left\{\left(X_{k}\right)_{i}\right\}_{i=a}^{b}$ for $a \geqslant 1$. By taking $b=n_{k}$, this proves $\mathbf{X}_{k}$ is stationary.

To show that the mean satisfies $\bar{X}=\bar{X}_{k}$ for all $0 \leqslant k \leqslant$ $d-1$, use induction. Here, the base case is trivially satisfied. So, write

$$
\begin{aligned}
n_{k+1} \bar{X}_{k+1} & =\sum_{i=1}^{n_{k+1}}\left(X_{k+1}\right)_{i} \stackrel{(1)}{=} \frac{1}{2} \sum_{i=1}^{n_{k} / 2}\left[\left(X_{k}\right)_{2 i-1}+\left(X_{k}\right)_{2 i}\right] \\
& =\frac{1}{2} n_{k} \bar{X}_{k}=n_{k+1} \bar{X}_{k},
\end{aligned}
$$

which provides the induction step.

Using Lemma 1 and Eq. (4) it is clear that any estimate of $\operatorname{var}(\bar{X})$ using $\sigma_{k}^{2} / n_{k}$ has truncation error given by

$$
e_{k} \equiv \frac{2}{n_{k}} \sum_{h=1}^{n_{k}-1}\left(1-\frac{h}{n_{k}}\right) \gamma_{k}(h)
$$

The next proposition is crucial: it says that if $\gamma_{0}(1), \gamma_{1}(1), \ldots, \gamma_{d-1}(1)$ are known, then the behavior of the truncation error $e_{k}$ is known. Corollary 1 will explain the details of this, but the main idea is that if $e_{k+1}-e_{k}$ is known for all $k$, then the values of $e_{k}$ are known up to a constant for all $k$.

Proposition 1. Suppose $2^{d} \geqslant 2$ is the number of observations, and $\sigma_{k}^{2}$ is finite for all $k \in\{0,1, \ldots, d-1\}$. Then, the rate of change of the truncation error $e_{k}$ is

$$
e_{k}-e_{k+1}=\frac{\gamma_{k}(1)}{n_{k}} \quad \text { for all } \quad 0 \leqslant k<d-1
$$

To prove the proposition, sum each side of Eq. (3) to get

$$
\sum_{h=1}^{n_{k+1}-1} \gamma_{k+1}(h)=\frac{1}{2} \sum_{h=1}^{n_{k}-1} \gamma_{k}(h)-\frac{1}{4}\left[\gamma_{k}(1)+\gamma_{k}\left(n_{k}-1\right)\right] .
$$

Similarly, sum Eq. (3):

$$
\sum_{h=1}^{n_{k+1}-1} h \gamma_{k+1}(h)=\frac{1}{4} \sum_{h=1}^{n_{k}-1} h \gamma_{k}(h)-\frac{n_{k}}{8} \gamma_{k}\left(n_{k}-1\right) .
$$

Plugging these equations into the definition of $e_{k}$ given in Eq. (5) and using $n_{k+1}=n_{k} / 2$, it is immediate that

$$
\begin{aligned}
e_{k+1} & =\frac{2}{n_{k+1}} \sum_{h=1}^{n_{k+1}-1}\left(1-\frac{h}{n_{k+1}}\right) \gamma_{k+1}(h) \\
& =\frac{4}{n_{k}} \sum_{h=1}^{n_{k+1}-1} \gamma_{k+1}(h)-\frac{8}{n_{k}^{2}} \sum_{h=1}^{n_{k+1}-1} h \gamma_{k+1}(h) \\
& \stackrel{(7)(8)}{=} e_{k}-\frac{\gamma_{k}(1)}{n_{k}} .
\end{aligned}
$$

The following corollary is the most important takeaway. It shows the effect of $\gamma_{k}(1)$ on the error of the estimate $\sigma_{k}^{2} / n_{k}$. Interestingly, this provides a proof of the behavior of the Flyvbjerg and Petersen [7] blocking method.

Corollary 1. Suppose $X_{1}, \ldots, X_{n}$ and $n=2^{d}>2$ are random variables from a weakly stationary sample with $\sigma_{k}^{2}$ finite for all $k \in\{0,1, \ldots, d-1\}$, and $i<j$ :

(1) If there exists $k \in \mathbb{N}$ such that for all $i \leqslant k \leqslant j$ either $\gamma_{k}(1)>0$ or $\gamma_{k}(1) \geqslant 0$ or $\gamma_{k}(1)=0$, then the sequence of errors $e_{k}$ is strictly decreasing or decreasing or constant on $i \leqslant k \leqslant j$, respectively.

(2) If there exists some $k \in\{0,1, \ldots, d-1\}$ such that the elements of $\mathbf{X}_{k}$ are uncorrelated, then the sequence of errors $e_{j}$ is constant, and $\sigma_{j+1}^{2}=\sigma_{j}^{2} / 2$ for all $j \geqslant k$.

Proof. Suppose the hypothesis is true and first let $\gamma_{k}(1)>$ 0 . That means Proposition 1 is true and there exist $k \in \mathbb{N}$ such that $\gamma_{k}(1)>0$ for all $i \leqslant k<j$. If $u, v \in\{i, i+1, \ldots, j+$ $1\}$ are distinct natural numbers, assume without loss of generality that $u<v$. By hypothesis, $n_{k}>0$ and $\gamma_{k}(1)>0$, and a sum of such terms must be positive. That means

$$
\begin{aligned}
0 & <\sum_{k=u}^{v-1} \frac{\gamma_{k}(1)}{n_{k}}=\frac{\gamma_{u}(1)}{n_{u}}+\frac{\gamma_{u+1}(1)}{n_{u+1}}+\cdots+\frac{\gamma_{v-1}(1)}{n_{v-1}} \\
& \stackrel{(6)}{=}\left(e_{u}-e_{u+1}\right)+\left(e_{u+1}-e_{u+2}\right)+\cdots+\left(e_{v-1}-e_{v}\right) \\
& =e_{u}-e_{v} .
\end{aligned}
$$

Now, by adding $e_{v}$ to each side of the inequality, the first part is proven. To obtain the result in the case $0 \leqslant \gamma_{k}(1)$, replace $<$ with $\leqslant$ in the argument above. The case $\gamma_{k}(1)=0$ is obtained by replacing $<$ with $=$.

Suppose $\delta_{i j}$ is the Kronecker delta. To obtain part 2, use induction: assume that the elements of $\mathbf{X}_{k}$ are uncorrelated. Then, the base case is trivially satisfied since all uncorrelated variables have zero covariance [3]. The induction step follows for $k+1$ since Eq. (3) says that $\gamma_{k+1}(i)=\delta_{i 0} \sigma_{k}^{2} / 2$. This proves $\gamma_{j}(1)$ is zero for all $j \geqslant k$, so the error is constant by what was proved above.

These results will be useful in the automation of the blocking method below. And, as stated, the corollary proves the behavior of the blocking method. But, experts will spot a problem: The sequence $e_{k}$ may be decreasing and eventually constant if the elements of $\mathbf{X}_{k}$ become uncorrelated. But, there is no guarantee that the variables become uncorrelated. However, existing users of the blocking method [7] know the variables do indeed become uncorrelated (and the constant from part 2 of the corollary is zero). But, so far this is not guaranteed. So, although our present results are promising and hint at the conclusions to come, a bit more work is 
required. The next part is technical, but the purpose is to obtain a decomposition of $\gamma_{k}(h)$ in Lemma 3. Start by fixing some $k \in\{0,1, \cdots\}$ and consider this interesting sequence of functions and its properties:

$$
f_{k}(i)= \begin{cases}i & \text { if } 0 \leqslant i \leqslant 2^{k} \\ 2^{k+1}-i & \text { if } 2^{k} \leqslant i \leqslant 2^{k+1} \\ 0 & \text { else. }\end{cases}
$$

Lemma 2. The sequence $\left\{f_{k}\right\}$ has the following nice properties:

(1) $f_{k}(i) \leqslant i$ for all $i \in \mathbb{N}$.

(2) $\sum_{i=1}^{2^{k+1}-1} f_{k}(i)=2^{2 k}$.

(3) $f_{k+1}(i)=f_{k}(i)+2 f_{k}\left(i-2^{k}\right)+f_{k}\left(i-2^{k+1}\right)$.

Proof. See the Appendix.

Lemma 3. Suppose $X_{1}, X_{2}, \ldots$ is a stationary time series and $h$ and $k$ are positive natural numbers, then,

$$
\gamma_{k}(h)=2^{-2 k} \sum_{i=1}^{2^{k+1}-1} f_{k}(i) \gamma\left[2^{k}(h-1)+i\right] .
$$

Proof. We prove the lemma by induction. Assume $k=1$ and write

$$
\begin{aligned}
\gamma_{1}(h) & \stackrel{(3)}{=} 2^{-2}\left(\gamma_{0}(2 h-1)+2 \gamma_{0}(2 h)+\gamma_{0}(2 h+1)\right) \\
& =2^{-2 k} \sum_{i=1}^{2^{k+1}-1} f_{k}(i) \gamma\left[2^{k}(h-1)+i\right] .
\end{aligned}
$$

Assume now that Eq. (10) is true for some $k \geqslant 1$ and write

$$
\begin{aligned}
2^{2(k+1)} \gamma_{k+1}(h) \stackrel{(3)}{=} & 2^{2 k}\left[\gamma_{k}(2 h-1)+2 \gamma_{k}(2 h)+\gamma_{k}(2 h+1)\right] \\
& \stackrel{(10)}{=} \sum_{i=1}^{2^{k+1}-1} f_{k}(i) \gamma\left[2^{k}(2 h-2)+i\right] \\
& +2 \sum_{i=1}^{2^{k+1}-1} f_{k}(i) \gamma\left[2^{k}(2 h-1)+i\right] \\
& +\sum_{i=1}^{2^{k+1}-1} f_{k}(i) \gamma\left[2^{k}(2 h)+i\right] \\
& \stackrel{(9)}{=} \sum_{i=1}^{2^{k+1+1}-1} \gamma\left[2^{k+1}(h-1)+i\right] \\
& \times\left[f_{k}(i)+2 f_{k}\left(i-2^{k}\right)+f_{k}\left(i-2^{k+1}\right)\right] \\
& \stackrel{\text { Lemma } 2}{=} \sum_{i=1}^{2^{k+1+1}-1} f_{k+1}(i) \gamma\left[2^{k+1}(h-1)+i\right] .
\end{aligned}
$$

In the third equality, the summation limits were shifted by $0,2^{k}$, and $2^{k+1}$, respectively; in addition, I used that $f_{n}(i-$ $\left.2^{j}\right)=0$ whenever $i \leqslant 2^{j}$ or $2^{k+1}+2^{j} \leqslant i$ from Eq. (9). This allowed to factor out the term $\gamma\left[2^{k+1}(h-1)+i\right]$.

Proposition 1 shows that $\gamma_{k}(1)$ is of special interest to us and therefore the following corollary is interesting.

Corollary 2. Suppose $X_{1}, X_{2}, \ldots$ is a stationary time series and $k$ is a positive natural number, then,

$$
\begin{aligned}
2^{2 k} \gamma_{k}(1)= & \gamma(1)+2 \gamma(2)+\cdots+2^{k} \gamma\left(2^{k}\right) \\
& +\left(2^{k}-1\right) \gamma\left(2^{k}+1\right)+\cdots+\gamma\left(2^{k+1}-1\right) .
\end{aligned}
$$

Proof. Use the previous lemma with $h=1$.

Using these results, everything is now set to finalize the investigation of $\gamma$ under blocking transformations. The following proposition may sound technical at first, but it will carry us all the way to the final proof of the blocking method.

Proposition 2. Assume that the stationary time series $X_{1}, X_{2}, \ldots$ has autocovariance $\gamma(h) \rightarrow 0$ as $h \rightarrow \infty$. Then, $\left\{\gamma_{k}\right\}_{k=1}^{\infty}$ converges uniformly to the zero function on $\mathbb{N}$.

Proof. Pick $\varepsilon>0$. By assumption $\gamma(i) \rightarrow 0$ as $i \rightarrow \infty$. So, there exists $I \in \mathbb{N}$ such that $\gamma(i)<\varepsilon / 2$ when $i \geqslant I$. Define $S=\left|\sum_{i=1}^{I} i \gamma(i)\right|$. Set $K=$ $\max \left\{\log _{2}(I),(1 / 2) \log _{2}(2 S / \varepsilon)\right\}$. Assume first that $h \geqslant 2$ and let $j \in \mathbb{N}$ be any natural number, then, by construction, if $k \geqslant K$ then we have

$$
\begin{gathered}
k \geqslant K \geqslant \log _{2} I \geqslant \log _{2} \frac{I}{h-1} \quad \text { only if } 2^{k}(h-1)+j \geqslant I \\
\text { only if } \gamma\left[2^{k}(h-1)+j\right]<\frac{\varepsilon}{2}
\end{gathered}
$$

since $\log _{2}$ is a monotonous function. Thus, by Lemma 3 and the triangular inequality

$$
\begin{aligned}
\left|\gamma_{k}(h)-0\right| & \leqslant 2^{-2 k} \sum_{j=1}^{2^{k+1}-1} f_{k}(j)\left|\gamma\left[2^{k}(h-1)+j\right]\right| \\
& \leqslant \frac{\varepsilon}{2} 2^{-2 k} \sum_{j=1}^{2^{k+1}-1} f_{k}(j)=\frac{\varepsilon}{2} 2^{-2 k} 2^{2 k}=\frac{\varepsilon}{2}<\varepsilon,
\end{aligned}
$$

where Lemma 2 was used in the third step. By construction, it is possible to assume $k \geqslant K \geqslant(1 / 2) \log _{2}(2 S / \varepsilon)$, so $\varepsilon / 2 \geqslant$ $2^{-2 k} S$. Assume now that $h=1$. Then, by Lemmas 2 and 3 and the triangular inequality

$$
\begin{aligned}
\left|\gamma_{k}(h)-0\right| & \leqslant 2^{-2 k}|\sum_{i=1}^{I} \underbrace{f_{i}(h)}_{\leqslant i} \gamma(i)|+2^{-2 k}\left|\sum_{i=I+1}^{2^{k+1}-1} f_{i}(h) \gamma(i)\right| \\
& <2^{-2 k} S+2^{-2 k} \frac{\varepsilon}{2}\left|\sum_{i=I+1}^{2^{k+1}-1} f_{i}(h)\right| \\
& <\frac{\varepsilon}{2}+2^{-2 k} \frac{\varepsilon}{2}|\underbrace{\sum_{i=1}^{2^{k+1}-1} f_{i}(h)}_{=2^{2 k}}| \leqslant \frac{\varepsilon}{2}+\frac{\varepsilon}{2}=\varepsilon
\end{aligned}
$$

which was required.

The blocking method follows immediately. The theorem says that end users can apply blocking transformations to get the truncation error smaller than every $\varepsilon>0$ if the time series is large enough.

Theorem 1 (The blocking method). Assume that the stationary time series $X_{1}, X_{2}, \ldots$ has autocovariance $\gamma(h) \rightarrow$ 0 as $h \rightarrow \infty$. Then, for every $\varepsilon>0$ there exists a natural number $K$ such that the magnitude of $e_{k}<\varepsilon$ if $K \leqslant k \leqslant d$ for the time series $X_{1}, X_{2}, \ldots, X_{2^{d}}$.

Proof. Suppose $\varepsilon>0$ is given. Since $\left\{\gamma_{k}\right\}_{k=1}^{\infty}$ converges uniformly and identically to zero on $\mathbb{N}$ by Proposition 2, there exists $K \in \mathbb{N}$ such that if $k \geqslant K$ then $\gamma_{k}<\varepsilon / 2$ on $\mathbb{N}$. 
Moreover, if $d \geqslant k$, then by the triangular inequality

$$
\begin{aligned}
\left|e_{k}\right| & \leqslant \frac{2}{n_{k}} \sum_{h=1}^{n_{k}-1}\left(1-\frac{h}{n_{k}}\right)\left|\gamma_{k}(h)\right| \leqslant \frac{2}{n_{k}} \sum_{h=1}^{n_{k}-1}\left|\gamma_{k}(h)\right| \\
& \leqslant \frac{\varepsilon}{n_{k}} \sum_{h=1}^{n_{k}-1} 1=\varepsilon \frac{n_{k}-1}{n_{k}}<\varepsilon,
\end{aligned}
$$

which is the theorem.

\section{AUTOMATING CALCULATIONS}

The previous section provided proof that if $X_{1}, X_{2}, \ldots$ is a stationary time series, then the error $\left\{\left|e_{k}\right|\right\}_{k=0}^{\infty}$ is a decreasing sequence that converges to zero whenever $\gamma(h) \rightarrow 0$ as $h \rightarrow \infty$. The next objective is to provide an algorithm that automates calculations. Users that just want to know the algorithm can skip to Sec. III B. Else, I introduce hypothesis testing and maximum likelihood estimation, which will be required to understand the results from the the present section.

Preliminaries 3. It is necessary to discuss which distribution the random variables and vectors represent: $\mathbf{A} \sim \alpha(\theta)$ indicates that the vector $\mathbf{A}$ is $\alpha$ distributed with parameter $\boldsymbol{\theta}$. In general, $\theta$ can have any dimension, and a $1 \times 1$ matrix or one-dimensional vector is a scalar. For example, let $\mathrm{N}(\mu, \Sigma)$ denote the multivariate normal distribution with expected value $\mu$ and covariance matrix $\Sigma$. If $\mathbf{Y} \sim \mathrm{N}(\mu, \Sigma)$ and $\Sigma$ is positive definite, then the probability density function (pdf) of $\mathbf{Y}$ is an $\mathbb{R}^{n} \rightarrow \mathbb{R}$ function

$$
f(\mathbf{y})=(2 \pi)^{-n / 2}|\Sigma|^{-1 / 2} \exp \left(-\frac{1}{2}(\mathbf{y}-\boldsymbol{\mu})^{\top} \Sigma^{-1}(\mathbf{y}-\boldsymbol{\mu})\right),
$$

where $|\cdot|$ denotes the determinant [10]. It turns out that $\mathbf{Y}$ is multivariate normal if and only if every linear combination of the elements of $\mathbf{Y}$ is normally distributed [11]. The components of $\mathbf{Y}$ are independent if and only if there exists $\sigma_{1}, \ldots, \sigma_{n}>0$ such that $\Sigma=\operatorname{diag}\left(\sigma_{1}, \ldots, \sigma_{n}\right)$ [10]. A random variable $X_{i} \sim \chi_{1}^{2}$ is $\chi^{2}$ distributed with 1 degree of freedom (later: dof) if it is the square of a standard normal random variable [3]. That means there exists a random variable $Z \sim \mathrm{N}(0,1)$ such that $X_{i}=Z^{2}$. A sum of $n$ independent $\chi^{2}$ random variables with 1 dof, $\sum_{i=1}^{n} X_{i}$, is $\chi^{2}$ distributed with $n$ dof and its pdf is

$$
g(x)=\frac{1}{2^{n / 2} \Gamma(n / 2)} x^{n / 2-1} e^{-x / 2} .
$$

Conveniently, if $\Sigma$ is invertible and $\left(Y_{1}, \ldots, Y_{n}\right)^{\top}=\mathbf{Y} \sim$ $\mathrm{N}(\boldsymbol{\mu}, \Sigma)$, then $(\mathbf{Y}-\boldsymbol{\mu})^{\top} \Sigma^{-1}(\mathbf{Y}-\boldsymbol{\mu}) \sim \chi_{n}^{2}$ [Proof. Write $\mathbf{Z}=\Sigma^{-1 / 2}(\mathbf{X}-\boldsymbol{\mu})$. What is the distribution of $\mathbf{Z}$ and $\mathbf{Z}^{\top} \mathbf{Z}$ ?] If $X \sim \chi_{v}^{2}$, the $100(1-\alpha)$ percentile is the value $q_{v}(1-$ $\alpha) \in \mathbb{R}$ such that $P\left[X>q_{v}(1-\alpha)\right]=\alpha \cdot \chi^{2}$ percentiles are tabulated in Table I. If $X_{1}, X_{2}, \ldots$ is a strictly stationary time series that is asymptotic uncorrelated such that $\operatorname{var}\left(X_{i}\right)<$ $\infty$ and $\lim _{n \rightarrow \infty} \operatorname{var}\left(\sum_{i=1}^{n} X_{i}\right)=\infty$, and $\left\langle\left|X_{i}\right|^{2+k}\right\rangle<\infty$ for some $k>0$, then [12] has proved that the central limit theorem holds [13].

The Fisherian approach to inference is the most common type of inference in natural sciences [14] and will be used in the following. Suppose there is a pdf $f(\mathbf{y} ; \boldsymbol{\theta})$ for $\mathbf{Y}$ that depends on a parameter $\theta$ and it is necessary to test whether there exists evidence that $\theta=\theta_{0}$ or if $\theta \neq \theta_{0}$ based on
TABLE I. $\chi^{2}$ 90, 95, and 99 percentiles: percentiles in the $\chi^{2}$ distribution for $1 \leqslant d-k \leqslant 48$, which suffices for any error

\begin{tabular}{|c|c|c|c|}
\hline$d-k$ & $q_{d-k}(0.99)$ & $q_{d-k}(0.95)$ & $q_{d-k}(0.9)$ \\
\hline 1 & 6.634897 & 3.841459 & 2.705543 \\
\hline 2 & 9.210340 & 5.991465 & 4.605170 \\
\hline 3 & 11.344867 & 7.814728 & 6.251389 \\
\hline 4 & 13.276704 & 9.487729 & 7.779440 \\
\hline 5 & 15.086272 & 11.070498 & 9.236357 \\
\hline 6 & 16.811894 & 12.591587 & 10.644641 \\
\hline 7 & 18.475307 & 14.067140 & 12.017037 \\
\hline 8 & 20.090235 & 15.507313 & 13.361566 \\
\hline 9 & 21.665994 & 16.918978 & 14.683657 \\
\hline 10 & 23.209251 & 18.307038 & 15.987179 \\
\hline 11 & 24.724970 & 19.675138 & 17.275009 \\
\hline 12 & 26.216967 & 21.026070 & 18.549348 \\
\hline 13 & 27.688250 & 22.362032 & 19.811929 \\
\hline 14 & 29.141238 & 23.684791 & 21.064144 \\
\hline 15 & 30.577914 & 24.995790 & 22.307130 \\
\hline 16 & 31.999927 & 26.296228 & 23.541829 \\
\hline 17 & 33.408664 & 27.587112 & 24.769035 \\
\hline 18 & 34.805306 & 28.869299 & 25.989423 \\
\hline 19 & 36.190869 & 30.143527 & 27.203571 \\
\hline 20 & 37.566235 & 31.410433 & 28.411981 \\
\hline 21 & 38.932173 & 32.670573 & 29.615089 \\
\hline 22 & 40.289360 & 33.924438 & 30.813282 \\
\hline 23 & 41.638398 & 35.172462 & 32.006900 \\
\hline 24 & 42.979820 & 36.415029 & 33.196244 \\
\hline 25 & 44.31410 & 37.65248 & 34.38159 \\
\hline 26 & 45.64168 & 38.88514 & 35.56317 \\
\hline 27 & 46.96294 & 40.11327 & 36.74122 \\
\hline 28 & 48.27824 & 41.33714 & 37.91592 \\
\hline 29 & 49.58788 & 42.55697 & 39.08747 \\
\hline 30 & 50.89218 & 43.77297 & 40.25602 \\
\hline 31 & 52.19139 & 44.98534 & 41.42174 \\
\hline 32 & 53.48577 & 46.19426 & 42.58475 \\
\hline 33 & 54.77554 & 47.39988 & 43.74518 \\
\hline 34 & 56.06091 & 48.60237 & 44.90316 \\
\hline 35 & 57.34207 & 49.80185 & 46.05879 \\
\hline 36 & 58.61921 & 50.99846 & 47.21217 \\
\hline 37 & 59.89250 & 52.19232 & 48.36341 \\
\hline 38 & 61.16209 & 53.38354 & 49.51258 \\
\hline 39 & 62.42812 & 54.57223 & 50.65977 \\
\hline 40 & 63.69074 & 55.75848 & 51.80506 \\
\hline 41 & 64.95007 & 56.94239 & 52.94851 \\
\hline 42 & 66.20624 & 58.12404 & 54.09020 \\
\hline 43 & 67.45935 & 59.30351 & 55.23019 \\
\hline 44 & 68.70951 & 60.48089 & 56.36854 \\
\hline 45 & 69.95683 & 61.65623 & 57.50530 \\
\hline 46 & 71.20140 & 62.82962 & 58.64054 \\
\hline 47 & 72.44331 & 64.00111 & 59.77429 \\
\hline 48 & 73.68264 & 65.17077 & 60.90661 \\
\hline
\end{tabular}
estimation with $\leqslant 10^{14}$ observations, at the three significance levels that performed best, $1-\alpha=0.99,0.95$, and 0.90 . See for example [3] for additional values.

the observations $\mathbf{Y}$. Let $\widehat{\theta}=\widehat{\theta}(\mathbf{Y})$ be an estimator for $\theta$. The hypothesis $\theta=\theta_{0}$ is called the null hypothesis, denoted $H_{0}$. It is common to pick $H_{0}$ such that the consequences of an incorrect test conclusion are minimized. The alternative hypothesis denoted by $H_{a}$ is typically the negation of $H_{0}$. 
Suppose there exists a $\mathbb{R}^{k} \rightarrow \mathbb{R}$ function $G(\widehat{\boldsymbol{\theta}})$ such that $G$ has known pdf $g$ whenever $H_{0}$ is true, then $G(\widehat{\boldsymbol{\theta}})$ is called a test statistic. The values of $G(\hat{\theta})$ that are sufficiently unlikely according to $g$ whenever $H_{0}$ is true are called the rejection region. And, if the estimated value $G(\widehat{\theta})$ is in the rejection region, $H_{0}$ is rejected in favor of $H_{a}$. Prior investigation $\alpha \in$ $(0,1)$ is chosen such that

$$
P\left(\text { Rejecting } H_{0} \mid H_{0} \text { is true }\right) \leqslant \alpha .
$$

Here, $P(A \mid B)$ denotes conditional probability. Since $g$ is known, this determines the size of the rejection region. It is convention to let $\alpha=0.05$ and say that the test result is significant if the estimated value $G(\widehat{\theta})$ is in the rejection region. It is possible to determine the largest value of $\alpha$ such that the test concludes that $H_{0}$ is false. This value is called the $p$ value, denoted $p$. The $p$ value is a measure of the probability that it is a mistake to reject $H_{0}$ in favor of $H_{a}$. The likelihood $L$ of $\boldsymbol{\theta}$ is the function $L(\theta)=f(\mathbf{Y} ; \boldsymbol{\theta})$. The estimator $\widehat{\theta}$ maximizing $L$ is called the maximum likelihood estimator, and is asymptotically multivariate normal distributed [11]. The estimator $\widehat{\gamma}_{k}(1)$ is a maximum likelihood estimator if $\mathbf{X}$ is multivariate normal. The bias of an estimator $\widehat{\theta}$ given a parameter $\theta$ is defined as $\operatorname{Bias}(\widehat{\theta} ; \theta)=$ $\langle\widehat{\theta}-\theta\rangle$ and measures how far from $\theta$ one can expect $\hat{\theta}$.

According to the variant of the central limit theorem introduced in Preliminaries 3, the elements of $\mathbf{X}_{j}$ are asymptotic multivariate normal if $\gamma(h) \rightarrow 0$ as $h \rightarrow \infty$ (in addition to some technical assumptions). This is because the elements of $\mathbf{X}_{k}$ are a mean of the elements of $\mathbf{X}$, which you can check. In that case, Preliminaries 3 say that $\widehat{\gamma}_{j}(1)$ is the maximum likelihood estimator of $\gamma_{j}(1)$. Hence, if $\widehat{\gamma}_{j} \equiv\left(\widehat{\gamma}_{j}(1), \ldots, \widehat{\gamma}_{d-1}(1)\right)$, then $\widehat{\gamma}_{j} \sim \mathrm{N}\left(\boldsymbol{\mu}_{j}, \Sigma_{j}\right)$ is asymptotic multivariate normal according to Preliminaries 3 . The idea is to find the first index $j$ such that $\gamma_{k}(1)=0$ for all $k \geqslant j$ because, by Corollary 1 , the error $e_{j}$ becomes constant and there is no reason to expect that $\sigma_{k}^{2} / n_{k}$ is a better estimate than $\sigma_{j}^{2} / n_{j}$ for any $k>j$. To test this, define

$$
M_{j}=\left(\widehat{\gamma}_{j}-\mu_{j}\right)^{\top} \Sigma_{j}^{-1}\left(\widehat{\gamma}_{j}-\mu_{j}\right) \sim \chi_{d-j}^{2} .
$$

Hence, $M_{j}$ has a known distribution (according to Preliminaries 3). This means that if $\boldsymbol{\mu}_{j}$ is evaluated in the case that $\gamma_{k}(1)=0$ for all $k \geqslant j$, it is a test statistic for the hypothesis test:

$$
\begin{aligned}
& H_{0}: \gamma_{k}(1)=0 \text { for all } k \geqslant j, \\
& H_{a}: \text { there exists } k \geqslant j \text { such that } \gamma_{k}(1) \neq 0 .
\end{aligned}
$$

The idea is to pick the smallest $j$ such that the hypothesis test finds no evidence for $H_{a}$ and take $\operatorname{var}(\bar{X})=\sigma_{j}^{2} / n_{j}$. Thus, an appropriate estimator is

$$
\widehat{\operatorname{var}}(\bar{X})=\widehat{\sigma}_{j}^{2} / n_{j}
$$

This works according to Preliminaries 3: Whenever $H_{0}$ is true, the distribution of $M_{j}$ is known $\left[\chi^{2}\right.$ with $d-j$ dof by Eq. (12)], so for all $j$ such that a sufficiently improbable value of $M_{j}$ is observed, the hypothesis test concludes that $H_{0}$ is false. However, once there is a $j$ such that $M_{j}$ is smaller than the $100(1-\alpha)$ percentile, there is no longer evidence for $H_{a}$ and the method concludes that $H_{0}$ is true,

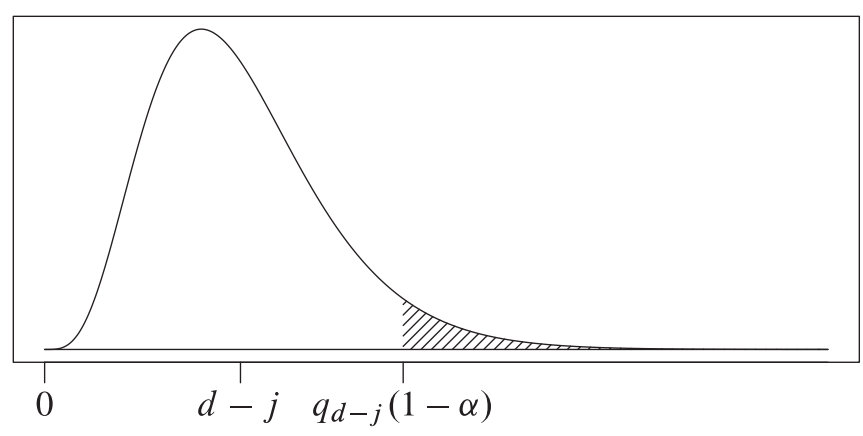

FIG. 1. Whenever $H_{0}$ is true, the pdf of $M_{j}$ is known and plotted above. The test concludes that $H_{0}$ is false if the observed value of $M_{j}$ is sufficiently unlikely. That is, if the observed value of $M_{j}$ is larger than $100(1-\alpha)$ percentile for a suitable $\alpha$; the shaded area represents a probability of $\alpha$. The value $d-j=\left\langle M_{j}\right\rangle$ is the expected value of $M_{j}$ whenever $H_{0}$ is true since then $M_{j} \sim \chi_{d-j}^{2}$ is $\chi^{2}$ distributed.

i.e., that the error becomes constant, and iterating further does not improve the estimate. See Fig. 1 for illustration. However, an expression of the covariance matrix $\Sigma_{j}$ has to be determined. In this paper, the following approximation will be used:

$$
\Sigma_{j} \simeq \operatorname{diag}\left(\sigma_{j}^{4} / n_{j}, \cdots, \sigma_{d-1}^{4} / n_{d-1}\right) .
$$

In the next section, we will see that the approximation is obtained by (a) considering the elements only up to leading order in $1 / n_{k}$ and (b) by setting all off-diagonal elements equal to zero. The benefit of the approximation is that inversion of $\Sigma_{j}$ is easy. It is possible to question the expected error of setting the off-diagonal elements equal to zero. But, the expected error is zero, as Proposition 6 explains. However, before proving that, more work is required. We end this section with a proposition which says that the estimators of any blocking method, $\bar{X}$ and $\widehat{\sigma}_{j}^{2} / n_{j}$, are necessarily asymptotically unbiased in the following sense.

Proposition 3. Assume that $X_{1}, X_{2}, \ldots$ is a weakly stationary time series. Then, $\bar{X}$ is an unbiased estimator of $\left\langle X_{i}\right\rangle$ for all $i \in \mathbb{N}$ and

$$
\operatorname{Bias}\left(\widehat{\sigma}_{j}^{2} / n_{j} ; \bar{X}\right)=-V(\bar{X}) / n_{j}-e_{j} \text { for all } j \geqslant 0 .
$$

If in addition $\gamma(h) \rightarrow 0$ as $h \rightarrow \infty$, then for every $\varepsilon>0$, there exists $K \in \mathbb{N}$ such that $\left|\operatorname{Bias}\left(\widehat{\sigma}_{K}^{2} / n_{K} ; \bar{X}\right)\right|<\varepsilon$.

Proof. Whenever $X_{1}, X_{2}, \ldots$ is a weakly stationary time series, there exists $\mu \in \mathbb{R}$ such that $\left\langle X_{i}\right\rangle=\mu$ for all $i \in \mathbb{N}$, so

$$
\langle\bar{X}\rangle=\left\langle\frac{1}{n} \sum_{i=1}^{n} X_{i}\right\rangle=\frac{1}{n} \sum_{i=1}^{n}\left\langle X_{i}\right\rangle=\frac{1}{n} n \mu=\left\langle X_{i}\right\rangle .
$$

To obtain the bias formula, use both the variance formula $\operatorname{var}(Y)=\left\langle Y^{2}\right\rangle-\langle Y\rangle^{2}(*)$ and $\sum_{i=1}^{n_{k}}\left(X_{k}\right)_{i}=n \bar{X}(\ddagger)$. Then, the following expression is obtained:

$$
\begin{aligned}
\left\langle\widehat{\sigma}_{K}^{2}\right\rangle & =\frac{1}{n_{K}} \sum_{i=1}^{n_{K}}\left\langle\left[\left(X_{K}\right)_{i}-\bar{X}\right]^{2}\right\rangle \\
& \stackrel{(\ddagger)}{=} \frac{1}{n_{K}} \sum_{i=1}^{n_{K}}\left[\left\langle\left(X_{K}\right)_{i}^{2}\right\rangle\right]+\left\langle\bar{X}^{2}\right\rangle-2\left\langle\bar{X}^{2}\right\rangle \stackrel{(*)}{=} \sigma_{K}^{2}-\operatorname{var}(\bar{X}) .
\end{aligned}
$$


Use Eq. (4) and write

$$
\begin{aligned}
\langle\widehat{\operatorname{var}}(\bar{X})\rangle & =\frac{\left\langle\widehat{\sigma}_{j}^{2}\right\rangle}{n_{j}}=\frac{\sigma_{j}^{2}}{n_{j}}-\frac{1}{n_{j}} \operatorname{var}(\bar{X}) \\
& =\operatorname{var}(\bar{X})-e_{j}-\frac{1}{n_{j}} \operatorname{var}(\bar{X}) .
\end{aligned}
$$

Subtract $\operatorname{var}(\bar{X})$ from each side of the equation, and the bias formula (16) is obtained.

For the third part, suppose that $\varepsilon>0$ is given. Since the hypothesis of Theorem 1 is true, there is a $K \in \mathbb{N}$ such that $\left|e_{k}\right|<\varepsilon / 2$ if $K \leqslant k \leqslant d$ for the time series $X_{1}, X_{2}, \ldots, X_{2^{d}}$. Pick $k=K$ and let $d>1+K+\log _{2} \operatorname{var}(\bar{X})-\log _{2} \varepsilon$. Because $d-K>1+\log _{2} \operatorname{var} \bar{X}-\log _{2} \varepsilon$,

$$
\frac{\operatorname{var}(\bar{X})}{n_{K}}=\operatorname{var}(\bar{X}) 2^{-(d-K)}<\operatorname{var}(\bar{X}) 2^{\log _{2}(\varepsilon)-\log _{2}(\bar{X})-1}=\frac{\varepsilon}{2} .
$$

Apply the triangular inequality and see

$$
\begin{aligned}
\left|\operatorname{Bias}\left(\widehat{\sigma}_{K}^{2} / n_{K} ; \operatorname{var}(\bar{X})\right)\right| & =\left|-\frac{\operatorname{var}(\bar{X})}{n_{K}}-e_{K}\right| \\
& \leqslant\left|\frac{\operatorname{var}(\bar{X})}{n_{K}}\right|+\left|e_{K}\right|<\frac{\varepsilon}{2}+\frac{\varepsilon}{2}=\varepsilon,
\end{aligned}
$$

which was required.

\section{A. Covariance matrix of $\widehat{\gamma}_{i}(h)$ and the matrix $\Sigma_{j}$}

It is necessary to compute the covariance matrix of the estimators $\widehat{\gamma}(1)$ because the estimator of the hypothesis test from the previous section depends on it. It is impractical to compute the covariance matrix directly. However, developing linear algebra for the task is a fruitful alternative. Two lemmas and two propositions are required. The idea is to lay the foundation to apply the following theorem from the theory of quadratic forms of random variables.

Preliminaries 4. Assume $\mathbf{Y} \sim \mathrm{N}(\boldsymbol{\mu}, \Sigma)$ with $\Sigma$ singular. If $A, B$ are symmetric $n \times n$ matrices and there exists some $n \times$ $r$ matrix $Q$ of rank $r$ such that $\Sigma=Q Q^{\top}$, then

$$
\operatorname{cov}\left(\mathbf{Y}^{\top} A \mathbf{Y}, \mathbf{Y}^{\top} B \mathbf{Y}\right)=2 \operatorname{Tr}(\Sigma A \Sigma B)+4 \boldsymbol{\mu}^{\top} A \Sigma B \boldsymbol{\mu} .
$$

For proof, see [15].

Consider now a lemma that contains all the information required about probability distributions:

Lemma 4. Assume $\mathbf{1}$ denotes the vector of ones, $\mathbf{X} \sim$ $\mathrm{N}\left(m \mathbf{1}, \sigma^{2} I_{n}\right)$ and $\mathbf{Y}=\mathbf{X}-\bar{X} \mathbf{1}$. Then, $\mathbf{Y}$ is multivariate normal with expected value $\boldsymbol{\mu}=\mathbf{0}$, and there exists some $n \times$ (n-1) matrix $Q$ of rank $n-1$ such that the covariance matrix $\Sigma_{\mathbf{Y}}=Q Q^{\top}$ and

$$
\Sigma_{\mathbf{Y}}=\frac{\sigma^{2}}{n}\left(n I_{n}-\mathbf{1 1}^{\top}\right) .
$$

Proof. First note that $Y_{i}$ is a linear combination of elements of $\mathbf{X}$ because $\mathbf{X}$ is multivariate normal, which means that $Y_{i}$ is univariate normal. This holds also for every linear combination of the elements of $\mathbf{Y}$, so $\mathbf{Y}$ is multivariate normal by Preliminaries 3. The expected value of $\mathbf{Y}$ is $\mathbf{0}$ since $\left\langle Y_{i}\right\rangle=\left\langle X_{i}-\bar{X}\right\rangle=m-m=0$. To get Eq. (20), notice that the covariance matrix of $\mathbf{X}$ is diagonal, which means that the elements of $\mathbf{X}$ are independent since $\mathbf{X}$ is multivariate normal, and if $\delta_{i j}$ denotes the Kronecker delta, then the elements of $\Sigma_{Y}$ are

$$
\begin{aligned}
\left(\Sigma_{\mathbf{Y}}\right)_{i j}= & \operatorname{cov}\left(Y_{i}, Y_{j}\right)=\operatorname{cov}\left(X_{i}-\bar{X}, X_{j}-\bar{X}\right) \\
= & \sigma^{2} \delta_{i j}-\frac{1}{n} \sum_{k=1}^{n} \operatorname{cov}\left(X_{i}, X_{k}\right) \\
& -\frac{1}{n} \sum_{k=1}^{n} \operatorname{cov}\left(X_{j}, X_{k}\right)+\operatorname{var} \bar{X} \\
= & \sigma^{2} \delta_{i j}-\frac{1}{n} \sum_{k=1}^{n} \sigma^{2} \delta_{i k}-\frac{1}{n} \sum_{k=1}^{n} \sigma^{2} \delta_{j k} \\
& +\frac{\sigma^{2}}{n}=\sigma^{2} \delta_{i j}-\frac{\sigma^{2}}{n}
\end{aligned}
$$

only if $\Sigma_{\mathbf{Y}}=\left(\sigma^{2} / n\right)\left(n I_{n}-\mathbf{1 1}^{\boldsymbol{\top}}\right)$. This proves that $\Sigma_{\mathbf{Y}}$ is symmetric. Note that $11^{\top} \mathbf{1}=n \mathbf{1}$, so $\mathbf{1}$ is an eigenvector of $\Sigma_{\mathbf{Y}}$ with eigenvalue 0 . Furthermore, if $k \in\{1,2, \ldots, n-1\}$ and $\mathbf{q}_{k}=\mathbf{e}_{k}-\mathbf{e}_{n}$, then

$$
\Sigma_{\mathbf{Y}} \mathbf{q}_{k} \stackrel{(21)}{=} \frac{\sigma^{2}}{n}\left[n \mathbf{q}_{k}-\mathbf{1}(\underbrace{\mathbf{1}^{\top} \mathbf{e}_{k}-\mathbf{1}^{\top} \mathbf{e}_{n}}_{=1-1=0})\right]=\sigma^{2} \mathbf{q}_{k},
$$

which proves that $\sigma^{2}$ is an eigenvalue of $\Sigma_{\mathbf{Y}}$ with multiplicity $n-1$. And since $\Sigma_{\mathbf{Y}}$ is symmetric, it has a spectral decomposition [16]:

$\Sigma_{\mathbf{Y}}=\sigma^{2} \sum_{k=1}^{n-1} \mathbf{q}_{k} \mathbf{q}_{k}^{\top}=\sigma^{2}\left[\begin{array}{lllll}\mathbf{q}_{1} & \mathbf{q}_{2} & \ldots & \mathbf{q}_{n-1}\end{array}\right]\left[\begin{array}{llll}\mathbf{q}_{1}^{\top} & \mathbf{q}_{2}^{\top} & \ldots & \mathbf{q}_{n-1}^{\top}\end{array}\right]^{\top}$.

So if $Q=\sigma\left[\begin{array}{llll}\mathbf{q}_{1} & \mathbf{q}_{2} & \ldots & \mathbf{q}_{n-1}\end{array}\right]$, then $Q$ is an $n \times(n-1)$ matrix and $\Sigma_{\mathbf{Y}}=Q Q^{\top}$. Moreover, according to the spectral theorem [16], the dimension of $\operatorname{span}\left\{\mathbf{q}_{1}, \ldots, \mathbf{q}_{n-1}\right\}$ equals the multiplicity of $\sigma^{2}$. That means the columns of $Q$ are $n-1$ linearly independent vectors, which also equals its rank.

Define transformations $S_{i}: \mathbb{R}^{n_{i}} \rightarrow \mathbb{R}^{n_{i}}$ and $T_{i}: \mathbb{R}^{n_{i-1}} \rightarrow$ $\mathbb{R}^{n_{i}}$ with standard matrices

$$
\begin{aligned}
S_{i} & =\left[\begin{array}{ccccc}
0 & 1 & 0 & 0 & 0 \\
0 & 0 & 1 & 0 & 0 \\
\vdots & \vdots & & \ddots & \\
0 & 0 & \cdots & 0 & 1 \\
0 & 0 & \cdots & \cdots & 0
\end{array}\right], \\
T_{i} & =\frac{1}{2}\left[\begin{array}{cccccc}
1 & 1 & 0 & 0 & \cdots & 0 \\
0 & 0 & 1 & 1 & \cdots & 0 \\
\vdots & \vdots & & \ddots & \ddots & \vdots \\
0 & 0 & 0 & 0 & 1 & 1
\end{array}\right] .
\end{aligned}
$$

According to Eq. (1), the matrices $T_{i}$ generate the observations $\mathbf{X}_{k}$ subject to $i$ blocking transformations by

$$
\mathbf{X}_{i}=T_{i} T_{i-1} \ldots T_{1} \mathbf{X}
$$


Using the matrices $\left\{S_{i}\right\}_{i=0}^{d-1}$ and $\left\{T_{i}\right\}_{i=1}^{d-1}$, define the $n \times n$ matrices $\left\{\Gamma_{i}\right\}_{i=0}^{d-1}$ by

$$
\Gamma_{i}=\frac{1}{2} \frac{1}{n_{i}} T_{1}^{\top} T_{2}^{\top} \ldots T_{i}^{\top}\left(S_{i}+S_{i}^{\top}\right) T_{i} \ldots T_{1} .
$$

According to the following proposition, these matrices are interesting because they generate the estimator $\widehat{\gamma}_{i}(1)$ from the vector $\mathbf{Y}$ whose probability distribution is multivariate normal.

Proposition 4. The matrices $\left\{\Gamma_{i}\right\}$ are symmetric. Hence, if $\mathbf{Y}=\mathbf{X}-\bar{X} \mathbf{1}$, then $\mathbf{Y}^{\top} \Gamma_{i} \mathbf{Y}$ is a quadratic form and $\mathbf{Y}^{\top} \Gamma_{i} \mathbf{Y}=$ $\widehat{\gamma_{i}}(1)$.

Proof. Fix $0 \leqslant i \leqslant d-1$. It is clear that $\Gamma_{i}$ is symmetric by construction:

$$
\begin{aligned}
\Gamma_{i}^{\top} & =\frac{1}{2} \frac{1}{n_{i}}\left(T_{1}^{\top} T_{2}^{\top} \ldots T_{i}^{\top}\left(S_{i}+S_{i}^{\top}\right) T_{i} \ldots T_{1}\right)^{\top} \\
& =\frac{1}{2} \frac{1}{n_{i}}\left(T_{i} \ldots T_{1}\right)^{\top}\left(S_{i}+S_{i}^{\top}\right)^{\top}\left(T_{1}^{\top} T_{2}^{\top} \ldots T_{i}^{\top}\right)^{\top}=\Gamma_{i} .
\end{aligned}
$$

That means $\mathbf{Y}^{\top} \Gamma_{i} \mathbf{Y}$ is a quadratic form. It remains to prove $\mathbf{Y}^{\top} \Gamma_{i} \mathbf{Y}=\widehat{\gamma}_{i}(1)$. First, use the definition of blocking transformation and that any real number equals it own transpose to obtain

$$
\begin{aligned}
n_{i} \mathbf{Y}^{\top} \Gamma_{i} \mathbf{Y} \stackrel{(24)}{=} & \frac{1}{2} \mathbf{Y}^{\top} T_{1}^{\top} \ldots T_{i}^{\top} S_{i} T_{i} \ldots T_{1} \mathbf{Y} \\
& +\frac{1}{2}\left[\mathbf{Y}^{\top} T_{1}^{\top} \ldots T_{i}^{\top} S_{i} T_{i} \ldots T_{1} \mathbf{Y}\right]^{\top} \\
= & {\left[T_{i} \ldots T_{1} \mathbf{Y}\right]^{\top} S_{i} T_{i} \ldots T_{1} \mathbf{Y} \stackrel{(23)}{=} \mathbf{Y}_{i}^{\top} S_{i} \mathbf{Y}_{i} . }
\end{aligned}
$$

Second, fix $1 \leqslant k \leqslant n_{i}$ and use induction to see $\left(\mathbf{Y}_{i}\right)_{k}=$ $\left(\mathbf{X}_{i}\right)_{k}-\bar{X}_{i}$. The base case is satisfied by hypothesis and the induction step follows by

$$
\begin{aligned}
\left(\mathbf{Y}_{i+1}\right)_{k} & \stackrel{(1)}{=} \frac{1}{2}\left[\left(\mathbf{Y}_{i}\right)_{2 k-1}+\left(\mathbf{Y}_{i}\right)_{2 k}\right] \\
& =\frac{1}{2}\left[\left(\mathbf{X}_{i}\right)_{2 k-1}-\bar{X}_{i}+\left(\mathbf{X}_{i}\right)_{2 k}-\bar{X}_{i}\right] \\
& \stackrel{(1)}{=}\left(\mathbf{X}_{i+1}\right)_{k}-2 \frac{1}{2} \bar{X}_{i} \stackrel{\text { Lemma }(1)}{=}\left(\mathbf{X}_{i+1}\right)_{k}-\bar{X}_{i+1},
\end{aligned}
$$

where Lemma 1 was used twice to get $\bar{X}_{i}=\bar{X}=\bar{X}_{i+1}$. Third, using the definition of the matrices $S_{i}$ from Eq. (22) notice that $S_{i}$ shifts the indices of vectors by one:

$$
\begin{aligned}
n_{i} \mathbf{Y}^{\top} \Gamma_{i} \mathbf{Y} & \stackrel{(25)}{=} \mathbf{Y}_{i}^{\top} S_{i} \mathbf{Y}_{i}=\mathbf{Y}_{i}^{\top}\left(S_{i} \mathbf{Y}_{i}\right) \\
& =\left(\left(\mathbf{Y}_{i}\right)_{1}, \cdots,\left(\mathbf{Y}_{i}\right)_{n_{i}}\right)^{\top}\left(\left(\mathbf{Y}_{i}\right)_{2}, \cdots,\left(\mathbf{Y}_{i}\right)_{n_{i}}, 0\right) \\
& =\sum_{h=1}^{n_{i}-1}\left(\mathbf{Y}_{i}\right)_{h+1}\left(\mathbf{Y}_{i}\right)_{h} \stackrel{(2)(26)}{=} n_{i} \widehat{\gamma}_{i}(1),
\end{aligned}
$$

using $\left(\mathbf{Y}_{i}\right)_{k}=\left(\mathbf{X}_{i}\right)_{k}-\bar{X}_{i}$ and the definition of $\widehat{\gamma}_{i}(1)$ in the final step.

Experts will immediately see that the above result is easily generalized to $\widehat{\gamma}_{k}(h)$ for any $h \geqslant 0$ by considering the operators $S_{k}^{h}$ by raising to a power $h \in \mathbb{Z}$. But, according to Proposition 1, it suffices to consider $h=1$. Some readers may later aim to compute the mean-squared error (MSE) of the blocking method estimator $\widehat{\operatorname{var}}(\bar{X})$. In doing so, they may want to repeat calculations for $h=0$ after reading Sec. III B. In the case of $h=1$, we have a technical lemma; the following three quantities determine the expression for the covariance matrix of $\widehat{\gamma}_{k}(1)$.

Lemma 5. If 1 denotes the vector of ones and $i \geqslant j$, then $\Gamma_{i}$ and $\Gamma_{j}$ constitute the following:

$$
\begin{aligned}
n_{i} \mathbf{1}^{\top} \Gamma_{i} \mathbf{1} & =n_{i}-1, \\
n^{2} n_{i} n_{j} \operatorname{Tr}\left[\Gamma_{i} \Gamma_{j}\right] & =\frac{1}{2} n_{i}^{2}\left(n_{i}-1\right), \\
2 n n_{i} n_{j} \mathbf{1}^{\top} \Gamma_{i} \Gamma_{j} \mathbf{1} & =2 n_{j}\left(n_{i}-1\right)-n_{i} .
\end{aligned}
$$

Proof. The following is used throughout: If $\left\{\mathbf{e}_{k}\right\}_{k=1}^{n_{i}}$ denotes the standard basis of $\mathbb{R}^{n_{i}}$, then according to Eq. (22),

$$
S_{i} \mathbf{e}_{k}=\left\{\begin{array}{ll}
\mathbf{0} & \text { if } k=1, \\
\mathbf{e}_{k-1} & \text { else }
\end{array} \text { and } S_{i}^{\top} \mathbf{e}_{k}= \begin{cases}\mathbf{0} & \text { if } k=n_{i}, \\
\mathbf{e}_{k+1} & \text { else. }\end{cases}\right.
$$

By multiplying $T_{i}$ by each vector from $\left\{\mathbf{e}_{k}\right\}_{k=1}^{n_{i}}$ and summing over $k$, it is clear that

$$
\begin{aligned}
& T_{i} \sum_{k=1}^{n_{i-1}} \mathbf{e}_{k}=T_{i} \mathbf{e}_{1}+T_{i} \mathbf{e}_{2}+T_{i} \mathbf{e}_{3}+\cdots+T_{i} \mathbf{e}_{n_{i-1}} \\
& \stackrel{(22)}{=} \frac{1}{2} \mathbf{e}_{1}+\frac{1}{2} \mathbf{e}_{1}+\frac{1}{2} \mathbf{e}_{2}+\cdots \frac{1}{2} \mathbf{e}_{n_{i}}=\sum_{k=1}^{n_{i}} \mathbf{e}_{k} .
\end{aligned}
$$

Write $\mathbf{1}$ as $\sum_{u=1}^{n} \mathbf{e}_{u}=\mathbf{1}$, and get the first equation:

$$
\begin{aligned}
n_{i} \mathbf{1}^{\top} \Gamma_{i} \mathbf{1} & =\frac{1}{2} \sum_{u=1}^{n} \mathbf{e}_{u}^{\top} T_{1}^{\top} T_{2}^{\top} \ldots T_{i}^{\top}\left(S_{i}+S_{i}^{\top}\right) T_{i} \ldots T_{1} \sum_{v=1}^{n} \mathbf{e}_{v} \\
& \stackrel{(28)}{=} \frac{1}{2} \sum_{u=1}^{n_{i}} \sum_{v=1}^{n_{i}} \mathbf{e}_{u}^{\top}\left(S_{i}+S_{i}^{\top}\right) \mathbf{e}_{v} \\
& =\frac{1}{2} \sum_{u=1}^{n_{i}} \sum_{v=2}^{n_{i}} \mathbf{e}_{u}^{\top} \mathbf{e}_{v-1}+\frac{1}{2} \sum_{u=1}^{n_{i}} \sum_{v=1}^{n_{i}-1} \mathbf{e}_{u}^{\top} \mathbf{e}_{v+1}=n_{i}-1,
\end{aligned}
$$

where orthonormality of $\left\{\mathbf{e}_{k}\right\}$ was used in the final step. Next, it is necessary to show $T_{i} T_{i}^{\top}=(1 / 2) I_{n_{i}}$ for all $1 \leqslant i \leqslant d-$ 1. To see this is true, write $T_{i}$ as a Kronecker product $T_{i}=$ $(1 / 2) I_{n_{i}} \otimes(1,1)$ and use the mixed product rule [17]:

$$
T_{i} T_{i}^{\top}=\frac{1}{4}\left(I_{n_{i}} \otimes(1,1)\right)\left(I_{n_{i}}^{\top} \otimes(1,1)^{\top}\right)=\frac{1}{4} \underbrace{I_{n_{i}}^{2}}_{I_{n_{i}}} \underbrace{(1,1)(1,1)^{\top}}_{=2} .
$$

Using this and working in a similar way as before, the following is obtained:

$$
2 n n_{i} n_{j} \mathbf{1}^{\top} \Gamma_{i} \Gamma_{j} \mathbf{1}=2 n_{i} n_{j}-n_{i}-2 n_{j}
$$

Prove now two more properties of $\left\{\mathbf{e}_{k}\right\}$ : First, see that if $M$ is any $n \times n$, then a diagonal element $m_{k k}=\mathbf{e}_{k}^{\top} M \mathbf{e}_{k}$, so $\operatorname{Tr}(M)=\sum_{k=1}^{n} \mathbf{e}_{k}^{\top} M \mathbf{e}_{k}$, as you can check. Second, if $M$ is a $n_{j+h} \times n_{j+h}$ matrix, then there is a real number $K \in \mathbb{R}$ such that

$$
\begin{aligned}
& \sum_{k=1}^{n_{j}-1} \mathbf{e}_{k}^{\top} T_{j+1}^{\top} \ldots T_{j+h}^{\top} M T_{j+h} \ldots T_{j+1} \mathbf{e}_{k+1} \\
& \quad=2^{-2 h} \sum_{k=1}^{n_{j+h}-1} \mathbf{e}_{k}^{\top} M \mathbf{e}_{k+1}+K \sum_{k=1}^{n_{j+h}} \mathbf{e}_{k}^{\top} M \mathbf{e}_{k} .
\end{aligned}
$$


Prove this by induction. If $h=1$, then

$$
\begin{aligned}
\sum_{k=1}^{n_{j}-1} & \mathbf{e}_{k}^{\top} T_{j+1}^{\top} M T_{j+1} \mathbf{e}_{k+1} \\
= & \frac{1}{4} \mathbf{e}_{1}^{\top} M \mathbf{e}_{1}+\frac{1}{4} \mathbf{e}_{1}^{\top} M \mathbf{e}_{2} \\
& +\cdots+\frac{1}{4} \mathbf{e}_{n_{j+1}-1}^{\top} M \mathbf{e}_{n_{j+1}}+\frac{1}{4} \mathbf{e}_{n_{j+1}}^{\top} M \mathbf{e}_{n_{j+1}} \\
& +\cdots 2^{-2} \sum_{k=1}^{n_{j+1}-1} \mathbf{e}_{k}^{\top} M \mathbf{e}_{k+1}+\frac{1}{4} \sum_{k=1}^{n_{j+1}} \mathbf{e}_{k}^{\top} M \mathbf{e}_{k},
\end{aligned}
$$

which proves the base case. To get the induction step, assume the hypothesis is true for $h$ then define the matrix $N=$ $T_{j+h+1}^{\top} M T_{j+h+1}$. This matrix is $n_{j+h} \times n_{j+h}$, so it is possible to use it in the place of the matrix $M$. Then, use the same procedure as before to prove the result for $h+1$.

To get the final equation from the lemma, use again that $T_{j} T_{j}^{\top}=2^{-1} I_{n_{j}}$, as well as cyclic permutation of the factors and write $\operatorname{Tr}\left[\Gamma_{i} \Gamma_{j}\right]$ in the following way:

$$
\begin{aligned}
4 n_{i} n_{j} \operatorname{Tr}\left[\Gamma_{i} \Gamma_{j}\right] \stackrel{(24)}{=} & 2^{-2 j} \operatorname{Tr}\left[T_{j+1}^{\top} \ldots T_{i}^{\top}\left(S_{i}+S_{i}^{\top}\right)\right. \\
& \left.\times T_{i} \ldots T_{j+1}\left(S_{j}+S_{j}^{\top}\right)\right] .
\end{aligned}
$$

Distribute the terms in the trace. One of the terms is $\operatorname{Tr}\left[T_{j+1}^{\top} \ldots T_{i}^{\top} S_{i} T_{i} \ldots T_{j+1} S_{j}^{\top}\right]$. To evaluate it, use what was just proven and write

$$
\begin{aligned}
& \sum_{k=1}^{n_{j}} \mathbf{e}_{k}^{\top} T_{j+1}^{\top} \ldots T_{i}^{\top} S_{i} T_{i} \ldots T_{j+1} S_{j}^{\top} \mathbf{e}_{k} \\
& \quad=\sum_{k=1}^{n_{j}-1} \mathbf{e}_{k}^{\top} T_{j+1}^{\top} \ldots T_{i}^{\top} S_{i} T_{i} \ldots T_{j+1} \mathbf{e}_{k+1} \\
& \stackrel{(30)}{=} 4^{-(i-j)} \sum_{k=1}^{n_{i}-1} \mathbf{e}_{k}^{\top} S_{i} \mathbf{e}_{k+1}+K \sum_{k=1}^{n_{i}} \underbrace{\mathbf{e}_{k}^{\top} S_{i} \mathbf{e}_{k}}_{\mathbf{e}_{k}^{\top} \mathbf{e}_{k-1}=0} .
\end{aligned}
$$

This term equals $4^{-(i-j)}\left(n_{i}-1\right)$ since $\mathbf{e}_{k}^{\top} S_{i} \mathbf{e}_{k+1}=\mathbf{e}_{k}^{\top} \mathbf{e}_{k}=1$. Make the replacement $S_{i} \mapsto S_{i}^{\top}$ throughout the above equation, in which case the term will evaluate to zero since $\mathbf{e}_{k}^{\top} S_{i}^{\top} \mathbf{e}_{k+1}=\mathbf{e}_{k}^{\top} \mathbf{e}_{k+2}=0$. The third and fourth terms from Eq. (31) are evaluated in a similar way. The sum of all four terms is $2 \times 4^{-(i-j)}\left(n_{i}-1\right)$, hence,

$$
\begin{aligned}
n^{2} n_{i} n_{j} \operatorname{Tr}\left[\Gamma_{i} \Gamma_{j}\right] & \stackrel{(31)}{=} 2 \frac{1}{4} 2^{-2 j} n^{2} 4^{-(i-j)}\left(n_{i}-1\right) \\
& =\frac{1}{2} n_{i}^{2}\left(n_{i}-1\right)
\end{aligned}
$$

which is the final part of the lemma.

The following proposition gives an expression for the elements of the covariance matrix of $\widehat{\gamma}_{i}(1)$.

Proposition 5. If there is some $m \in \mathbb{R}$ such that the vector $\mathbf{X} \sim \mathrm{N}\left(m \mathbf{1}, \sigma^{2} I_{n}\right)$, then the expected value of $\widehat{\gamma}_{i}(1)$ is $-\sigma_{i}^{2}\left(n_{i}-1\right) / n_{i}^{2}$. Furthermore, the covariance matrix of $\left(\widehat{\gamma}_{0}(1), \ldots, \widehat{\gamma}_{d-1}(1)\right)^{\top}$ has elements

$\operatorname{cov}\left(\widehat{\gamma}_{i}(1), \widehat{\gamma}_{j}(1)\right)=2\left(\frac{\sigma_{i} \sigma_{j}}{n_{i} n_{j}}\right)^{2}\left[1+\left(n_{i}-1\right)\left(\frac{1}{2} n_{i}^{2}-n_{j}\right)\right]$

whenever $0 \leqslant j \leqslant i \leqslant d-1$.
Proof. Assume $0 \leqslant j \leqslant i \leqslant d-1$. To obtain the expectation, use the defining equation (2) and notice that the elements of $\mathbf{X}_{i}$ are independent by hypothesis, so

$n_{i}\left\langle\widehat{\gamma_{i}}(1)\right\rangle$

$$
=\sum_{j=1}^{n_{i}-1} \underbrace{\left\langle\left(\mathbf{X}_{i}\right)_{j}\left(\mathbf{X}_{i}\right)_{j+1}\right\rangle}_{\gamma_{i}(1)+m^{2}=0+m^{2}}+\underbrace{\left\langle\bar{X}^{2}\right\rangle}_{\substack{\sigma_{i}^{2} \\ n_{i} \\ n^{2}}}-\underbrace{\left\langle\left[\left(\mathbf{X}_{i}\right)_{j}+\left(\mathbf{X}_{i}\right)_{j+1}\right] \bar{X}\right\rangle}_{2\left(m^{2}+\sigma_{i}^{2} / n_{i}\right)},
$$

and the first part is proven. Assume now that $\mathbf{Y}=\mathbf{X}-\bar{X} \mathbf{1}$. To get the covariance matrix, note that by Lemma $4, \mathbf{Y}$ is multivariate normal with expected value $\mathbf{0}$ and there exists a $n \times(n-1)$ matrix $Q$ of rank $n-1$ such that the covariance matrix $\Sigma_{\mathbf{Y}}=Q Q^{\top}$. According to Proposition $4, \Gamma_{i}$ and $\Gamma_{j}$ are symmetric, so according to Preliminaries 4 ,

$$
\begin{aligned}
\operatorname{cov}\left(\widehat{\gamma_{i}}(1), \widehat{\gamma}_{j}(1)\right) & =\operatorname{cov}\left(\mathbf{Y}^{\top} \Gamma_{i} \mathbf{Y}, \mathbf{Y}^{\top} \Gamma_{j} \mathbf{Y}\right) \\
& \stackrel{(19)}{=} 2 \operatorname{Tr}\left(\Sigma_{\mathbf{Y}} \Gamma_{i} \Sigma_{\mathbf{Y}} \Gamma_{j}\right) .
\end{aligned}
$$

Recall that it is possible to cyclic permute the elements of a trace and that $\Gamma_{i}=\Gamma_{j}^{\top}$, and $\operatorname{Tr}(M)=\operatorname{Tr}\left(M^{\top}\right)$ and $\operatorname{Tr}\left(\mathbf{1}^{\top} M \mathbf{1}\right)=\mathbf{1}^{\top} M \mathbf{1}$ (since it is a real number) for all square matrices $M$. Using this and Lemma 4, write $\left(n^{2} / \sigma^{4}\right) \operatorname{Tr}\left(\Sigma_{\mathbf{Y}} \Gamma_{i} \Sigma_{\mathbf{Y}} \Gamma_{j}\right)$ in the following way:

$$
\begin{aligned}
\frac{n^{2}}{\sigma^{4}} \operatorname{Tr}\left(\Sigma_{\mathbf{Y}} \Gamma_{i} \Sigma_{\mathbf{Y}} \Gamma_{j}\right)= & n^{2} \operatorname{Tr}\left(\Gamma_{i} \Gamma_{j}\right)+\mathbf{1}^{\top} \Gamma_{i} \mathbf{1 1}^{\top} \Gamma_{j} \mathbf{1} \\
& -2 n \mathbf{1}^{\top} \Gamma_{i} \Gamma_{j} \mathbf{1} .
\end{aligned}
$$

To complete the proof, use now Lemma 5 and that $n_{i}=n / 2^{i}$. Furthermore, since the elements of $\mathbf{X}$ are independent, $\sigma_{j}^{2}=$ $\sigma^{2} / 2^{j}$ by Corollary 1 . Thus,

$$
\begin{aligned}
\operatorname{Tr}\left(\Sigma_{\mathbf{Y}} \Gamma_{i} \Sigma_{\mathbf{Y}} \Gamma_{j}\right)= & \frac{\sigma^{4}}{n^{2}}\left[n_{i} n_{j}\right]^{-1}\left[\frac{1}{2} n_{i}^{2}\left(n_{i}-1\right)+\left(n_{i}-1\right)\left(n_{j}-1\right)\right. \\
& \left.+n_{i}-2 n_{j}\left(n_{i}-1\right)\right] \\
= & \left(\frac{\sigma_{i} \sigma_{j}}{n_{i} n_{j}}\right)^{2}\left[1+\left(n_{i}-1\right)\left(\frac{1}{2} n_{i}^{2}-n_{j}\right)\right] .
\end{aligned}
$$

Multiply each side of the equation by 2 and recall Eq. (32) above, which proves the proposition true.

As we discussed, the diagonal elements of the covariance matrix of $\widehat{\gamma_{i}}(1)$ are of special interest to us, and they are given by the following corollary.

Corollary 3. Assume there is some $m \in \mathbb{R}$ such that the vector $\mathbf{X} \sim \mathrm{N}\left(m \mathbf{1}, \sigma^{2} I_{n}\right)$. Then, the variance of $\widehat{\gamma}_{j}(k)$ is exactly

$$
\operatorname{var}\left(\widehat{\gamma}_{j}(1)\right)=\left(\frac{\sigma_{j}}{n_{j}}\right)^{4}\left[2+n_{j}\left(n_{j}-1\right)\left(n_{j}-2\right)\right],
$$

whenever $0 \leqslant j \leqslant d-1$.

It remains to determine the error of discarding the offdiagonal elements of $\Sigma_{j}$ in Eq. (15). In order to understand how the error may influence the calculation, recall that the purpose of finding $\Sigma_{j}$ is to evaluate $M_{j}$. If we form a diagonal matrix $\Sigma_{j}^{\prime}$ that contains the diagonal elements of $\Sigma_{j}$ and 
make the approximation

$$
M_{j}^{\prime} \equiv\left(\widehat{\gamma}_{j}-\mu_{j}\right)^{\top} \Sigma^{\prime}{ }_{j}^{-1}\left(\widehat{\gamma}_{j}-\mu_{j}\right),
$$

then the expected error $\left\langle M_{j}-M_{j}^{\prime}\right\rangle$ is zero according to the following proposition:

Proposition 6. If $E_{j}=M_{j}-M_{j}^{\prime}$, then $\left\langle E_{j}\right\rangle=0$.

Proof. Set $G_{j}=\widehat{\gamma}_{j}-\mu_{j}$ and use the definition of $\Sigma_{j}$ to write $\Sigma_{j}$ in a convenient way:

$$
\Sigma_{j}=\left\langle\left(\widehat{\gamma}_{j}-\boldsymbol{\mu}_{j}\right)\left(\widehat{\gamma}_{j}-\boldsymbol{\mu}_{j}\right)^{\top}\right\rangle=\left\langle G_{j} G_{j}^{\top}\right\rangle .
$$

In this notation we can write $M_{j}=G_{j}^{\top} \Sigma_{j}^{-1} G_{j}$ and similarly for $M_{j}^{\prime}$. Since $E_{j}$ is a $1 \times 1$ matrix and using that the trace is invariant under cyclic permutation of its elements $(\dagger)$,

$$
\begin{aligned}
E_{j} & =\operatorname{Tr} E_{j}=\operatorname{Tr}\left[M_{j}-M_{j}^{\prime}\right]=\operatorname{Tr}\left[G_{j}^{\top}\left(\Sigma_{j}^{-1}-\Sigma_{j}^{\prime-1}\right) G_{j}\right] \\
& \stackrel{(\dagger)}{=} \operatorname{Tr}\left[\left(\Sigma_{j}^{-1}-\Sigma_{j}^{-1}\right) G_{j} G_{j}^{\top}\right] .
\end{aligned}
$$

It follows directly from the definition of matrix trace and linearity of expected value that if $A$ is a (nonrandom) matrix then $\left\langle\operatorname{Tr} A G_{j} G_{j}^{\top}\right\rangle=\operatorname{Tr}\left\langle A G_{j} G_{j}^{\top}\right\rangle=\operatorname{Tr} A\left\langle G_{j} G_{j}^{\top}\right\rangle$ [10]. That means

$$
\left\langle E_{j}\right\rangle=\operatorname{Tr}\left[\left(\Sigma_{j}^{-1}-\Sigma_{j}^{\prime-1}\right)\left\langle G_{j} G_{j}^{\top}\right\rangle\right] \stackrel{(33)}{=} \operatorname{Tr}\left[\left(I-\Sigma_{j}^{\prime-1} \Sigma_{j}\right)\right] .
$$

Define $m=d-j$, then $\Sigma_{j}$ and $\Sigma_{j}^{\prime}$ are $m \times m$ matrices. Also, note that by assumption, $\Sigma_{j}^{\prime}$ is the diagonal matrix consisting of diagonal elements of $\Sigma_{j}$. That means (1) the inverse of $\Sigma_{j}^{\prime}$ is a diagonal matrix, and (2) it has diagonal elements that are the multiplicative inverse of the diagonal elements of $\Sigma_{j}$. So,

$$
\left(\Sigma_{j}^{\prime-1}\right)_{i k}=\left(\Sigma_{j}\right)_{i k}^{-1} \delta_{i k} .
$$

Consequently, each diagonal element of $\Sigma_{j}^{\prime-1} \Sigma_{j}$ equals 1 because

$$
\begin{aligned}
\left(\Sigma_{j}^{\prime-1} \Sigma_{j}\right)_{i i} & =\sum_{k=1}^{m}\left(\Sigma_{j}^{\prime-1}\right)_{i k}\left(\Sigma_{j}\right)_{k i}=\sum_{k=1}^{m}\left(\Sigma_{j}\right)_{i k}^{-1} \delta_{i k}\left(\Sigma_{j}\right)_{k i} \\
& =\left(\Sigma_{j}\right)_{i i}^{-1}\left(\Sigma_{j}\right)_{i i}=1 .
\end{aligned}
$$

So, since the matrix trace is the sum of all the diagonal elements,

$$
\operatorname{Tr} \Sigma_{j}^{\prime-1} \Sigma_{j}=\sum_{i=1}^{m}\left(\Sigma_{j}^{\prime-1} \Sigma_{j}\right)_{i i} \stackrel{(35)}{=} \sum_{i=1}^{m} 1=m
$$

and has the consequence that

$$
\left\langle E_{j}\right\rangle \stackrel{(34)(33)}{=} \underbrace{\operatorname{Tr} I}_{=m}-\operatorname{Tr} \Sigma_{j}^{\prime-1} \Sigma_{j} \stackrel{(36)}{=} m-m=0,
$$

which is the proposition.

Applying this proposition in combination with Corollary 3 to leading order in $1 / n_{k}$ provides justification for the use of $\operatorname{diag}\left(\sigma_{j}^{4} / n_{j}, \cdots, \sigma_{d-1}^{4} / n_{d-1}\right)$ in the place of $\Sigma_{j}$ in computation of $M_{j}$.

\section{B. Algorithm}

By summarizing the results from the two previous sections, the following is clear:
Theorem 2. If $X_{1}, X_{2}, \ldots$ is a strictly stationary time series such that $\gamma(h) \rightarrow 0$ as $h \rightarrow \infty$ with $\lim _{n \rightarrow \infty} \operatorname{var}\left(\sum_{i=1}^{n} X_{i}\right)=\infty$ and $\left\langle\left|X_{i}\right|^{2+a}\right\rangle<\infty$, for some $a>0$, then $M_{j}$ is a test statistic that is asymptotic $\chi_{d-j}^{2}$ distributed under the hypothesis $\gamma_{k}(1)=0$ for all $k \geqslant j$. The rejection region includes all values $M_{j}$ larger than $q_{d-j}(1-\alpha)$ for all $1 \leqslant j \leqslant d-1$.

The above theorem outlines the algorithm. Form a vector $\mathbf{X}$ consisting of $2^{d}$ observations. The algorithm proceeds as follows: Compute $\widehat{\sigma}_{i}^{2}$ and $\widehat{\gamma}_{i}(1)$ for $\mathbf{X}_{i}$ for each $i \in\{0,1, \ldots, d-1\}$. Then, form $M_{i}$ for all $i \in$ $\{0,1, \ldots, d-1\}$ using the estimates $\widehat{\sigma}_{i}^{2}$ and $\widehat{\gamma}_{i}(1)$. Using the results from the two previous sections, $M_{j}$ becomes

$$
M_{j}=\sum_{k=j}^{d-1} \frac{n_{k}\left[\left(n_{k}-1\right) \widehat{\sigma}_{k}^{2} /\left(n_{k}^{2}\right)+\widehat{\gamma}_{k}(1)\right]^{2}}{\widehat{\sigma}_{k}^{4}} .
$$

Pick some significance level $\alpha$. It is convention in inference to let $\alpha=0.05$, but it is possible to pick some other value. Then, compare $M_{k}$ to $q_{d-k}(1-\alpha)$ for all $k$. Choose the smallest $k$ such that $M_{k} \leqslant q_{d-k}(1-\alpha)$. Using this $k$, make the final estimate for the variance $\operatorname{var}(\bar{X})=\widehat{\sigma}_{k}^{2} / n_{k}$.

The method has built in safety features (see Fig. 6). This is necessary because the method may operate without supervision. If the conditions above are not met, the method may fail. In case this happens, it is necessary to present a warning to the end user or application so they can take necessary action. Recall the conditions for the method (see Theorem 2): (i) the time series is strictly stationary, (ii) $\gamma(h) \rightarrow 0$ as $h \rightarrow$ $\infty$, and (iii) the $\chi^{2}$ approximation works. Therefore, if the method does not conclude that $H_{0}$ is true for any $k \leqslant d-1$, one of these are false, and the fault is caught with an if test. The conditions (i) and (ii) are either present or not by construction and, as such, end users will know whether these are satisfied or not. However, condition (iii) can fail if there are little data available. So, the bottom line is that the end user should check whether conditions (i) and (ii) are satisfied in order to guarantee that the method works reliably. If the end user is unsure if the time series is stationary, there exist statistical tests for stationary, such as the Dickey-Fuller test, which can be used. It is usually easy to check condition (ii) by estimating the autocovariance matrix by $\widehat{\gamma}(h)$ using a suitably small chunk of the data. See Fig. 6 for sample code of one implementation, or use flow chart of Fig. 2 for an overview.

The method is asymptotically unbiased in the way one would expect according to Proposition 3, which also gives an equation for the bias. Using that and Eq. (4) gives

$$
\begin{aligned}
\operatorname{Bias}(\widehat{\operatorname{var}}(\bar{X}) ; \operatorname{var}(\bar{X})) & \stackrel{(16)}{=}-\frac{\operatorname{var}(\bar{X})}{n_{K}}-e_{K} \\
& \stackrel{(4)}{=}-\operatorname{var}(\bar{X})\left(1+\frac{1}{n_{K}}\right)+\frac{\sigma_{K}^{2}}{n_{k}} .
\end{aligned}
$$




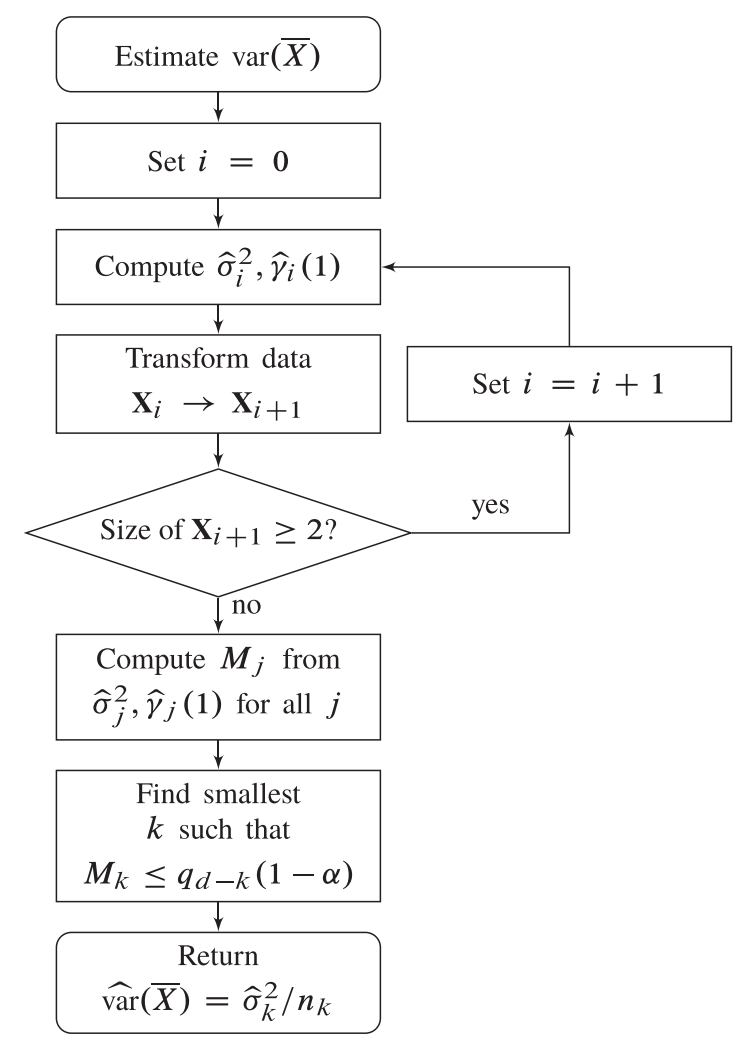

FIG. 2. Flow chart of algorithm. The idea is to return the estimate of $\operatorname{var}(\bar{X})$, as $\widehat{\sigma}_{k}^{2} / n_{k}$ for the smallest value of $k$ such that there is no evidence that $\gamma_{j}(1) \neq 0$ for $j>k$. This is sensible because then, according to Corollary 1 , there is no reason to believe that error $e_{k}$ is reduced by further iterations of the method.

By estimating each quantity separately, this is the resulting estimator of the bias of $\widehat{\operatorname{var}}(\bar{X})$ given $\operatorname{var}(\bar{X})$ :

$$
\widehat{\operatorname{Bias}} \stackrel{(37)}{=}-\widehat{\operatorname{var}}(\bar{X})\left(1+\frac{1}{n_{K}}\right)+\frac{\widehat{\sigma}_{K}^{2}}{n_{K}} \stackrel{(14)}{=}-\frac{\widehat{\operatorname{var}}(\bar{X})}{n_{K}} .
$$

The estimator can be used to survey the performance of the method if bias is critical to the application. In addition, it turns out to be relatively easy to compute the variance of the estimator $\widehat{\operatorname{var}}(\bar{X})$. Summing the bias squared and the variance of an estimator yields the mean-squared error (MSE) [3].

Preliminaries 5. MSE is a risk function corresponding to the expected value of the squared error loss, and one of the most popular measures of the performance of an estimator. According to [3], if $\widehat{\theta}$ is any estimator and $\theta$ a parameter, then

$$
\operatorname{MSE}(\widehat{\theta} ; \theta)=\left\langle(\widehat{\theta}-\theta)^{2}\right\rangle=\operatorname{Bias}^{2}(\widehat{\theta} ; \theta)+\operatorname{var}(\widehat{\theta}) .
$$

Since an expression of the bias is available, it remains to determine the variance of $\widehat{\operatorname{var}}(\bar{X})$. Working in a similar way as in Sec. III A, by first writing $\widehat{\sigma}_{K}^{2}$ as a quadratic form and then applying the result of Preliminaries 4, the following expression is obtained:

$$
\operatorname{var}\left(\widehat{\sigma}_{K}^{2}\right)=2\left(\frac{\sigma_{K}^{2}}{n_{K}}\right)^{2}\left(n_{K}-1\right) .
$$

It is possible to estimate each quantity of the equation separately, obtaining the following estimator of the MSE of $\widehat{\operatorname{var}}(\bar{X})$ given $\operatorname{var}(\bar{X})$ :

$$
\widehat{\operatorname{MSE}} \stackrel{(38)}{=}[\widehat{\operatorname{var}}(\bar{X})]^{2} \frac{2 n_{K}-1}{n_{K}^{2}} .
$$

It is now straightforward to implement these self-evaluation features in your estimation program. In the final part of this section, the announced upper bound on the computational complexity is derived.

An upper bound of the computational complexity of the method is $12 n$. Consider the sample code in Fig. 6. The only contributions at order $n$ are from the while loop. At iteration number $i$, the while loop can be computed using exactly $6 n_{i}$ floating point operations. Using geometric series, the total floating point operations are

$$
\text { cost }=\sum_{j=0}^{d-1} 6 n_{j}=6 \times 2^{d} \sum_{j=0}^{d-1} 2^{-j}=12(n-1) .
$$

For time consuming computations that require multithread computing or time series so large that it comes in chunks, this bound can be reduced to $n+O(1)$ as will be shown in Sec. III D, but first consider these test results of the present implementation.

\section{Test results}

The method validation uses autoregressive models because Wold decomposition justifies their use in modeling stationary time series [6]. Moreover, $\operatorname{var}(\bar{X})$ can be be computed exactly for autoregressive models. This makes them ideal for our purpose.

Preliminaries 6. An autoregressive model of order $p$ denoted $\operatorname{AR}(p)$ is a stochastic process $\left\{X_{t}\right\}_{t=1}^{\infty}$ such that

$$
X_{t}=\phi_{1} X_{t-1}+\phi_{2} X_{t-2}+\cdots+\phi_{p} X_{t-p}+\varepsilon_{t}
$$

for $\phi_{i} \in \mathbb{R}$ and random variables $\varepsilon_{t}$ that are independent, identically distributed with zero expected value and constant variance $\sigma^{2}$ for all $t$. The autoregressive models used have orders 1 and 2, they have autocovariance in closed form, and are stationary [6]. For all stationary processes, $\operatorname{var}(\bar{X})$ is given by the autocovariance $\gamma$, and for the $\operatorname{AR}(p)$-processes of interest to us, $\gamma$ is determined by the polynomial $P(z)=1-$ $\phi_{1} z-\phi_{2} z^{2}-\cdots-\phi_{p} z^{p}$. The autoregressive model is said to be causal if all the roots $z_{i}$ of $P(z)$ satisfy $\left|z_{i}\right|>1$.

Causal AR(1) and AR(2) process are easily parameterized to be asymptotic uncorrelated and stationary [6]. Two tests of the algorithm are presented. First, 6080 causal random $\mathrm{AR}(1)$ and $\mathrm{AR}(2)$ processes were generated. According to Preliminaries 6 , this means the exact value of $\operatorname{var}(\bar{X})$ can be computed from the autoregressive coefficients $\phi_{i}$ for each of the $\operatorname{AR}(p)$ processes. The relative error squared, $\epsilon^{2}$, converged to zero as a function of $n / \tau$. Here, $\tau$ denotes the time constant of the autocorrelation function $[\tau$ is the smallest integer such that $|\gamma(h)| \leqslant \gamma(0) e^{-1}$ for all $\left.h \geqslant \tau\right]$. Gamma regression is suitable because the observations of $\epsilon^{2}$ are independent, identically gamma distributed, and the model is $\log \left(\epsilon^{2}\right)=$ 
TABLE II. Regression summary for the $\operatorname{AR}(p)$ processes: regression table for the AR(1) process (left) and AR(2) (right). If $\epsilon$ denotes expected relative error, the model was $\log \left(\epsilon^{2}\right)=\beta_{0}+\beta_{1} \log (n / \tau)$. The regression family is taken to be gamma, and fitted by maximum likelihood estimation using iterative reweighted least squares. The estimated values of $\beta_{j}$ are given above along with standard errors. The $p$ values are given for the null hypothesis that $\beta_{j}=0$. Deviances explained are $50.65 \%$ and $65.39 \%$ for the AR(1) and AR(2) on 6078 degrees of freedom, respectively.

\begin{tabular}{lrrrrrrrr}
\hline \hline & \multicolumn{3}{c}{$\operatorname{AR}(1)$} & & \multicolumn{3}{c}{$\operatorname{AR}(2)$} \\
\cline { 2 - 3 } \cline { 6 - 8 } & Estimate & Std error & $p$ value & & Estimate & Std error & $p$ value \\
\hline$\beta_{0}$ & 0.7402 & 0.2592 & 0.00431 & & 2.4566 & 0.0991 & $<10^{-16}$ \\
$\beta_{1}$ & -0.5202 & 0.0271 & $<10^{-16}$ & & -0.7022 & 0.0108 & $<10^{-16}$ \\
\hline \hline
\end{tabular}

$\beta_{0}+\beta_{1} \log (n / \tau)$. The expected relative error squared is

$$
\epsilon^{2}=e^{\beta_{0}}\left(\frac{n}{\tau}\right)^{\beta_{1}}
$$
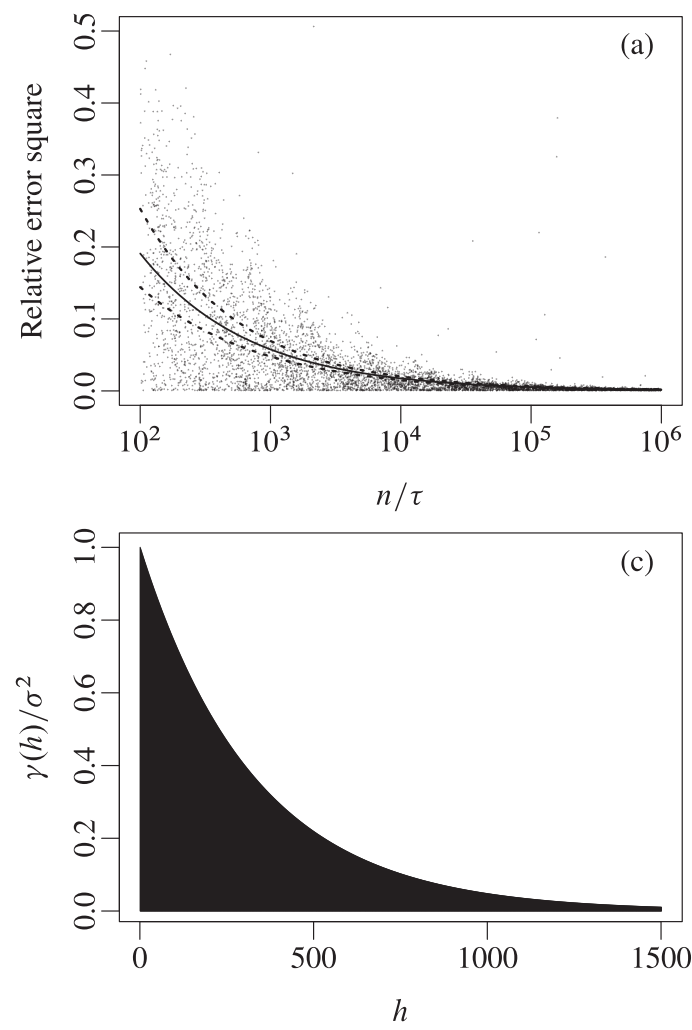

Maximum likelihood estimates of $\beta_{j}$ and standard errors are given for the causal AR(1) and AR(2) processes in Table II. The table shows that if there are very little data available (say $n=\tau$ ), then the relative error $\epsilon=0.7402^{1 / 2}=0.861$ and $2.4566^{1 / 2}=1.567$ for the $\mathrm{AR}(1)$ and $\mathrm{AR}(2)$ processes, respectively. It is also evident that the convergence rate of the $\mathrm{AR}(2)$ processes are faster than the $\mathrm{AR}(1)$ processes. Among the processes, the type of autocovariance was the main differentiator of the $\operatorname{AR}(p)$ processes. For $p=1$, the autocorrelation is of the form $\gamma(h) / \sigma^{2}=\phi^{-h}$, while in the case $p=2$, there exist $z \in \mathbb{C}$ and $a, b, c \in \mathbb{R}$ such that $\gamma(h) / \sigma^{2}=$ $a|z|^{-h} \cos (h b+c)$. Furthermore, no effect is found of the sampling distribution $(p \geqslant 0.34, \mathrm{t}$ test), and therefore the difference in $\epsilon^{2}$ between the $\operatorname{AR}(1)$ and $\operatorname{AR}(2)$ experiments is attributed to the $\gamma(h)$ according to Preliminaries 2. A plot of the regression analysis is given in Fig. 3 and for the regression summaries, see Table II.

Second, two standard textbook physics applications were studied. The variance of the mean energy was estimated using the Flyvbjerg and Petersen [7] blocking method and the automated blocking method. The estimates were compared with dependent bootstrapping using geometric simulation
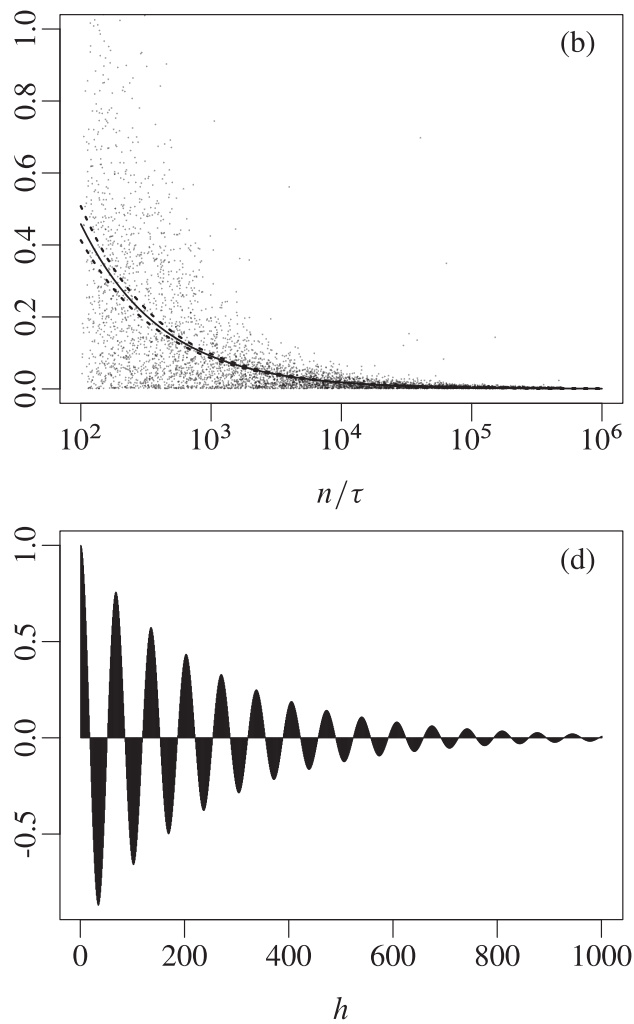

FIG. 3. Relative error squared of two autoregressive models versus observations per time autocorrelation time constant. There is exponential convergence rate for two common correlation structures in natural sciences. In the tail of the distribution, there are outlayers that failed the probabilistic test. The outlayers are not of major concern because (1) they are rare and (2) induced mistakes are small in magnitude. (a), (c) Represent test results for an AR(1) time series with an autocorrelation that is positive with exponential decay, typical of Metropolis-type Markov chains, where it is expected that the observations correlate positively. The process was distributed Gamma(1,1). (b), (d) Represent test results for an AR(2) process. The autocorrelation has exponential decay, but oscillates. The process was distributed multivariate standard normal. It was found that the method was insensitive to the distribution of the observations $(p \geqslant 0.34)$. Consequently, the difference in behavior of the method is attributed to $\gamma(h)$, as explained by Corollary 1 . The expected relative error squared $\epsilon^{2}$ was modeled by gamma regression, $\log \left(\epsilon^{2}\right)=\beta_{0}+\beta_{1} \log (n / \tau)$. Deviance explained was $50.65 \%$ and $65.39 \%$ for $\operatorname{AR}(1)$ and $\operatorname{AR}(2)$ on 6078 degrees of freedom, respectively. Dashed lines give $95 \%$ confidence intervals of the expected relative error squared. The plots indicate that it is reasonable to expect the first digit of the method was correct for some $n \gtrsim 20 \tau$, and two digits correct for some $n \gtrsim 25000 \tau$. 

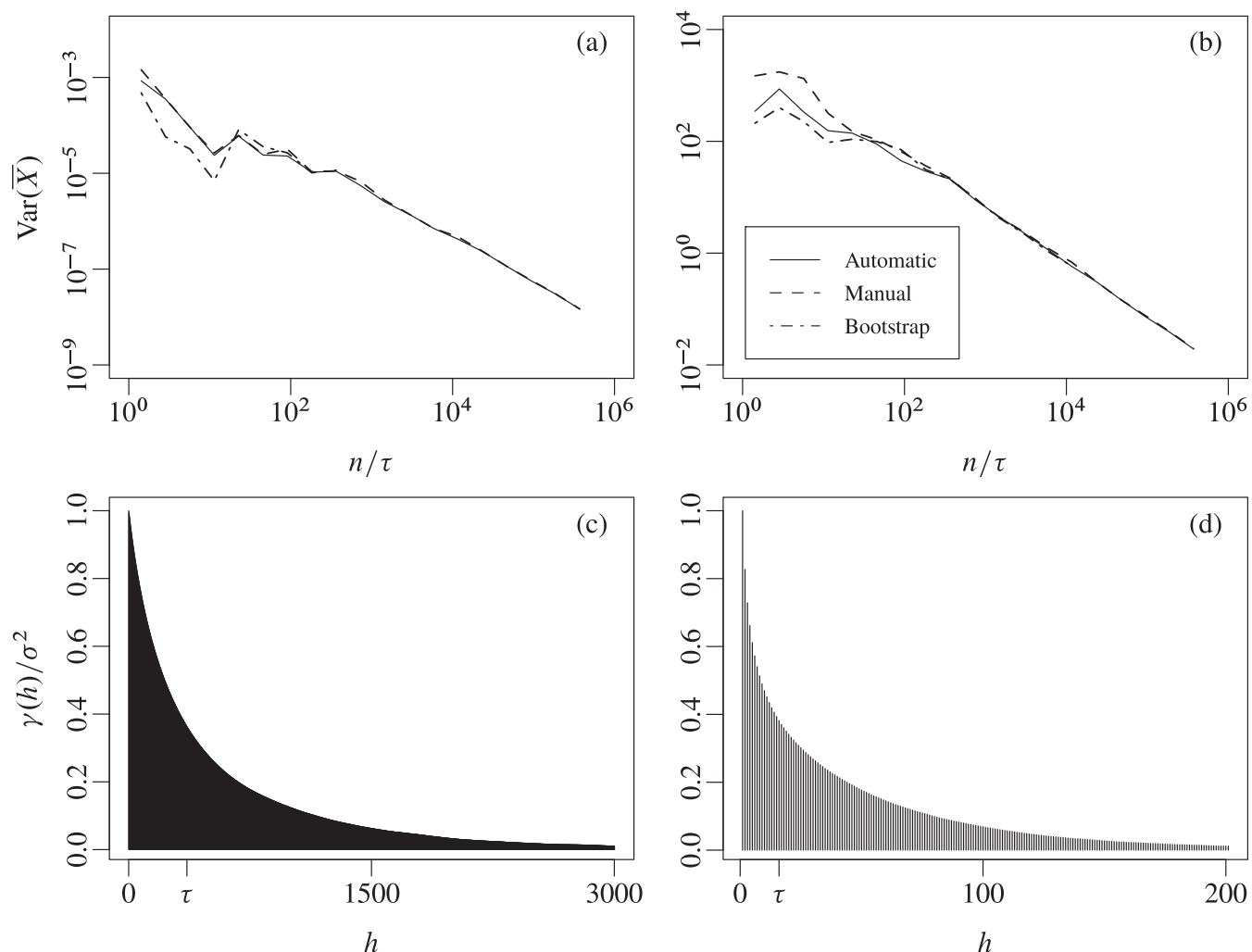

FIG. 4. Case study of two textbook physics applications for mean variance estimation. The variance of the mean energy was estimated using manual and automatic Blocking methods and compared with dependent bootstrapping using geometric simulation. The estimates are plotted in (a) and (b), while the autocorrelation is depicted in (c) and (d). (a), (c) Represent test results for a two electron quantum dot with trail energy of a Slater-Jastrow-type state function for a theory of a harmonic oscillator potential with Coulomb repulsion $(\omega=1)$. The importance sampling/Hastings-Metropolis theorem implementation implies acceptance rate $>99.999$. The autocorrelation time constant was $\tau=360$. It is clear that $\operatorname{var}(\bar{X})=1.46 \times 10^{-8}$ at $10^{8}$ samples according to all three models, and by the above discussion, the error of the variance estimate is correct to the second digit. (b), (d) Represent test results for the Ising model for a $20 \times 20$ grid of spins with periodic boundary conditions at temperature $T=2.4$. The energy was sampled from a Boltzman distribution at significance level $95 \%$ according to a $\chi^{2}$ test using a Metropolis-type Markov chain. The autocorrelation time constant was measured at $\tau=16$, so at $10^{6} \operatorname{samples} \operatorname{var}(\bar{X})=1.49 \times 10^{-1}$ according to all three methods, and there is reason to believe that the first two digits are correct.

type [18]. In either application, the autocorrelation functions bore resemblance of the AR(1) autocorrelation (in light of Corollary 1). The first application was an $n$-electron quantum dot with trail energy of a Slater-Jastrow-type state function for a theory of a harmonic oscillator potential with Coulomb repulsion. The angular frequency was $\omega=1$. Importance sampling/Hastings-Metropolis theorem was used together with a Fokker-Plank-type prior [19]. The implementation has acceptance rate $>99.999 \%$ for each proposed state. The autocorrelation time constant was $\tau=360$, and the time until the observations were close to uncorrelated was $h \approx 4 \tau$. The second application was an implementation of the Ising model using a $20 \times 20$ grid of spins with periodic boundary conditions at temperature $T=2.4$ [20]. The energy was sampled from a Boltzman distribution at significance level $\alpha=0.05$ according to a $\chi^{2}$ goodness-of-fit test [3] using a stationary, time-reversible Markov chain constructed using the Metropolis algorithm [21]. The autocorrelation time constant was measured to be $\tau=16$. Figure 4 plots the results.

\section{Multithread computing and memory limitations}

If the time series is sufficiently large, it is common to store the time series in smaller chunks, rather than in one file, or in memory all at once. Such can happen if the computing facility memory is smaller than the time series, or the application generating the time series runs on multiple threads. This is typically the case when the time series is generated by a Markov chain on multithread clusters. As shown above, it is possible to reduce the size of the data by applying blocking transformations on each chunk until the chunks are small enough to be imported onto a single node or personal computer. Suppose the amount of memory that can be allocated on this node is $2^{d}$ real numbers.

Assume now that the total length of the time series is $n=2^{D}$, divided into $2^{k}$ smaller chunks of length $2^{D-k}$. Let $\mathbf{X}$ denote any such vector containing a chunk of the time series. It is important that within such a chunk, the order of the observations is preserved. Now on the chunk, apply blocking transformations $D-d$ times and form $\mathbf{X}_{D-d}=$ $T_{D-d} T_{D-d-1} \ldots T_{1} \mathbf{X}$ where $T_{i}$ is defined in Eq. (22). The size of $\mathbf{X}_{D-d}$ is exactly $2^{D-k} / 2^{D-d}=2^{d-k}$. The same procedure is executed on each of the $2^{k}$ chunks, and thus the total data are of all the transformed chunks is $2^{k} 2^{d-k}=2^{d}$, as required. On the parent node, or personal computer doing the final estimate, write the data to memory by concatenating 
The time series can be split into $2^{k}$ chunks of length $2^{D-k}$ :

$$
\begin{aligned}
& X_{1}, X_{2}, \cdots, X_{2^{D-k}}, \underbrace{X_{2^{D-k}+1}, \cdots, X_{2^{D-k+1}}}_{\text {chunk number } i \equiv \mathbf{X}}, \cdots, X_{2^{D}} \\
& \begin{array}{l}
\text { (Step 1) Transform chunk } i \text { by: } \\
\qquad \mathbf{X}_{D-d}=T_{D-d} \cdots T_{2} T_{1} \mathbf{X}
\end{array} \\
& \mathbf{Y}_{i} \equiv \mathbf{X}_{D-d} \quad \text { (Step 2) Repeat for all } i \\
& (\mathbf{Y}_{1}, \mathbf{Y}_{2}, \cdots, \underbrace{\left., \mathbf{Y}_{i}, \cdots, \mathbf{Y}_{2^{k}}\right)^{\top}}_{\begin{array}{c}
\text { Transformed } \\
\text { chunk } i
\end{array}}
\end{aligned}
$$

FIG. 5. In the case that the time series is too large for memory, or is so large that it is not saved in a single file, it is possible to reduce the size of each chunk of the time series by repeated use of the matrices $T_{j}$. This is convenient in the case that the time series is generating by a multithreaded program. The procedure is as follows: Choose one of the chunks of the time series, number $i$. (Step 1) Transform chunk number $i$ by applying the matrix $T_{D-d} T_{D-d-1} \ldots T_{2} T_{1}$. Define $Y_{i}$ to be the result of this transformation. (Step 2) Repeat for all $j \neq i$. (Step 3) Concatenate all the chunks after they have been formed into one long vector $\left(\mathbf{Y}_{1}, \mathbf{Y}_{2}, \ldots, \mathbf{Y}_{2^{k}}\right)^{\top}$. This vector will now have size $2^{d}$, thus is small enough to (Step 4) be handled on a single node by using the algorithm of Fig. 2. See Sec. IIID for more details including the definitions of the numbers $D, d$, and $k$.

each of the $2^{d-k}$ blocks end to end into a long vector of size $2^{d}$, then perform the ordinary algorithm as it is given in Fig. 2. Figure 5 provides an overview of the procedure.

The computational cost of this is low. Performing the transformations $T_{i}$ as it is done in the code of Fig. 6 requires precisely $n_{i-1}$ floating point operations, as you can check. So, the total number of floating point operations is computed using geometric series:

$$
\sum_{j=1}^{D-d} n_{j-1}=\sum_{j=0}^{D-d-1} 2^{D-k-j}=\frac{2^{D}-2^{d}}{2^{k-1}} .
$$

According to Eq. (41), it is necessary to add the 12(2d -1$)$ floating point operations that the parent node must spend at the end, so the total cost is bounded above by

$$
\begin{aligned}
\operatorname{cost} & \stackrel{(43)}{\leqslant} \underbrace{2^{D}}_{=n} \frac{1}{2^{k-1}}+2^{d}(12-\underbrace{\frac{1}{2^{k-1}}}_{\leqslant 1}) \\
& \leqslant n+O(1) \quad \text { as } n \rightarrow \infty .
\end{aligned}
$$

\# data vector must be of size $2^{\wedge} d$ for some integer $d$ $\mathrm{X}=$ loadtxt ("data.txt")

$\mathrm{n}=\operatorname{len}(\mathrm{X}) ; \mathrm{d}=\log 2(\mathrm{n}) ; \mathrm{mu}=\operatorname{mean}(\mathrm{X}) ; \mathrm{i}=0$

$\mathrm{s}, \operatorname{gamma}=\operatorname{zeros}(\mathrm{d}), \operatorname{zeros}(\mathrm{d})$

\# Chi-square percentiles. More values in appendix

$\mathrm{q}=\operatorname{array}([6.634897, \ldots, 50.892181])$

\# Get autocovariance and variance for all X_i

while $\mathrm{n}>=2$ :

\# estimate variance and autocovariance of $X_{-} i$

$\mathrm{x}=\mathrm{X}-\mathrm{mu}$

$\operatorname{gamma}[i]=\operatorname{sum}(x[1: n] * x[\theta:(n-1)]) / n$

$s[i]=\operatorname{sum}(x * 2) / n$

\# perform blocking transformation

$y=\operatorname{zeros}(n / 2)$

for $j$ in arange $(\theta, n / 2)$ :

$y[j]=0.5 *(X[2 * j]+X[2 * j+1])$

$\mathrm{X}=\mathrm{y} ; \mathrm{n}=\mathrm{n} / 2 ; \mathrm{i}=\mathrm{i}+1$

\# Generate the test statistic $M_{-} j$

$\mathrm{M}=\operatorname{zeros}(\mathrm{d})$

for $j$ in arange $(\theta, d)$ :

$\mathrm{n}=2 * *(\mathrm{~d}-\mathrm{j})$

$M[j]=n *((n-1) * s[j] / n * * 2+\operatorname{gamma}[j]) * * 2 / s[j] * * 2$

\# elements reversed twice such cumsum is correct

$\mathrm{M}=\operatorname{cumsum}(\mathrm{M}[::-1])[::-1]$

\# Determine the smallest $\mathrm{k}$ such that H_Q is true

for $\mathrm{k}$ in arange $(\theta, \mathrm{d})$ :

if $(\mathrm{M}[\mathrm{k}]<\mathrm{q}[\mathrm{k}])$ :

break

if $(\mathrm{k}>=\mathrm{d}-1)$ :

print "Warning: Add more data."

\# and the answer is

$\mathrm{nk}=2 * *(\mathrm{~d}-\mathrm{k})$

answer $=\mathrm{s}[\mathrm{k}] / \mathrm{nk}$

FIG. 6. PYTHON implementation of the algorithm. The code is purposefully verbose to aid implementation in languages of lower level of abstraction, such as $\mathrm{C}$. In practice, the implementation can be optimized and shrunk to about 10-15 lines of code. The most recent implementations for PYTHON, $\mathrm{C}++$, and $\mathrm{R}$ are available (see [8]).

The reason it is possible to rejoin the time series by putting it end to end is the same reason dependent bootstrapping works: As long as the chunks are large enough, the resampling of putting the observations end to end does not change $\gamma$. See for example [18,22]

Analogously, the total mean can also be computed in chunks since it splits up into a mean of means. Define mean of chunk number $j$ by $\widehat{\mu}_{j}=\left(1 / 2^{D-k}\right) \sum_{i=(j-1) 2^{D-k}}^{j 2^{D-k}} X_{i}$, then write

$$
\bar{X}=\frac{1}{2^{D}} \sum_{i=1}^{2^{D}} X_{i}=\sum_{j=1}^{2^{k}} \frac{1}{2^{D}} \underbrace{\sum_{i=(j-1) 2^{D-k}}^{j 2^{D-k}}}_{2^{D-k} \widehat{\mu}_{j}} X_{i}=\frac{1}{2^{k}} \sum_{j=1}^{k} \widehat{\mu}_{j} .
$$

That implies the total mean of the time series is just the mean of all the means. All in all, there is no problem in splitting the whole time series in chunks since both statistics of interest are recovered at the end.

If the data are generated by a program, it is a time saver to compute the estimates on each thread at the same time the 
program is generating data. If you choose to do so, precision is maximized by making the chunks as large as possible. Working in this way saves considerable time because then the data do not have to be read back into memory for postprocessing later.

\section{DISCUSSION}

This study provides the following four new contributions: (a) rigorous proof of the Flyvbjerg and Petersen [7] blocking method conforming to the standard of modern mathematics. The results give prospects for new research with relevance to any blocking method. (b) An automated blocking method is provided. It works for a variety of autocovariance functions. (c) Autoregressive models were chosen to provide error estimates. These account for error due to both (i) the method and (ii) the sampling. (d) Integration of the blocking method for multithread computing or extremely large time series. The contributions include proof of the behavior of the method. Furthermore, Proposition 1 outlines an approach to (i) estimate the standard error more efficiently and (ii) provide economical error estimates, both in terms of computation simplicity and in precision. This method is simple to explain and implement (requires no more than 10-20 lines of code), and will appeal to those using the Flyvbjerg and Petersen [7] blocking method, because it works under more general conditions and maintains the simplicity of the original method.

Several authors have attempted to give justification for the use of the blocking methods. Best known is the work of Flyvbjerg and Petersen [7] providing motivational mathematics to explain the idea of Blocking transformations for standard error estimation. They claim that there exists "an obvious fixed point" but gives no proof [7]. For mathematically interested readers, this can present a distraction since fixed points of any function $T_{j}: A \rightarrow B$ are defined when $A=B[4,23,24]$. In this context, $A \neq B$ since $A=\mathbb{R}^{n_{i}}$ and $B=\mathbb{R}^{n_{i} / 2}$ (see Sec. I). Thus, in mathematical sense, there is no fixed point present. Instead, the justification given in the results is the following: For the blocking method, the variables subject to $k$ blocking transformations $\mathbf{X}_{k}$ form a stationary time series if $\mathbf{X}$ is stationary. This means that it is possible to express the error $e_{k}$ as a function of $\gamma_{k}(h)$. From this and the transformation properties of the $\gamma_{j}(h)$, it follows that the behavior of the truncation error is given by $\left\{\gamma_{k}(1)\right\}$. See Proposition 1. This may come as a surprise because this implies that the behavior of the method is determined by the set of $\left\{\gamma_{k}(1)\right\}_{k=0}^{d-1}$. Flyvbjerg and Petersen [7] appear unaware of this because they state that the blocking method converges if $\gamma(h) \propto 1 / h$ [7], which does not capture the essence, as was proved in Theorem 1. In fact, their blocking method works whenever $X_{1}, X_{2}, \ldots$ is asymptotic uncorrelated, as Theorem 1 makes precise.

Proposition 1 proves that the blocking method is applicable under more general conditions than assumed by Flyvbjerg and Petersen [7]. First, because $\gamma(h)$ need not be proportional to $1 / h$ [note Proposition 1 , which places no restriction on $\gamma(h)$, although finite variance is required]. Therefore, $\gamma(h)$ can have any shape. Second, Flyvbjerg and Petersen [7] constrain $\gamma(h)$, exactly $n$ degrees of freedom (since $\gamma$ is a function $\gamma:\{0,1, \ldots, n-1\} \rightarrow \mathbb{R})$, while the results only constrain $\left\{\gamma_{k}(1)\right\}$, exactly $\log _{2}(n)=d$ degrees of freedom. Theorem 1 may also have theoretical interest in statistical mathematics. The sum $s_{k}=\sum_{h=1}^{n_{k}-1}\left(1-h / n_{k}\right) \gamma_{k}(h)$, for $k=0$, appears frequently in the study of time series [6], Proposition 1 and Lemma 1 together imply that $\left(2 / n_{k}\right) s_{k}$ is determined up to a constant ${ }^{1}$ by the set $\left\{\gamma_{k}(1)\right\}$. Moreover, according to Theorem $1, \gamma_{k}(h)$ converges uniformly to zero on $\mathbb{N}$. In this way, blocking transformations are intimately linked to $s_{k}$. This provides interesting prospects for further work: for physics, it is possible to provide realistic error estimates and improve the method substantially if $\left\{\gamma_{k}(1)\right\}$ is estimated more accurately [since $e_{k}=\left(2 / n_{k}\right) s_{k}$ ]. However, elegant solutions probably require non-Fisherian statistics. Perhaps Bayesian statistics can be used because estimation is difficult for large $k$ simply because when $k$ is large, then the sample size $n_{k}$ available for estimation is small. For example, a suitable shrinkage estimator may be particularly useful. See, for example, Stein's phenomenon [25] and applications such as those by Schäfer and Strimmer [26]. Another proposed application of Proposition 1 is the proof of Corollary 1, which explains the behavior of the blocking method [7], and why the automatic blocking method works. The corollary shows that the estimates $\operatorname{var}(\bar{X})$ improve with each blocking transformation, until (i) the variables $\mathbf{X}_{k}$ become uncorrelated or (ii) there exist $j$ such that the covariances $\gamma_{k}(1)=0$ for all $k \geqslant j$. In case (i) the truncation error $e_{k}=0$ since if the components of $\mathbf{X}_{k}$ are uncorrelated, then $\operatorname{cov}\left(\left(\mathbf{X}_{k}\right)_{i},\left(\mathbf{X}_{k}\right)_{j}\right)=\sigma_{k}^{2} \delta_{i j}$ by definition, so $\gamma_{k}(h)=0$ for $h \geqslant 1$ and, hence,

$$
e_{k}=\frac{2}{n_{k}} \sum_{h=1}^{n_{k}-1}\left(1-\frac{h}{n_{k}}\right) \underbrace{\gamma_{k}(h)}_{=0}=0 .
$$

Proposition 2 strengthens this statement to include case (ii) because it shows that $\gamma_{k}$ converges uniformly and identically to zero on $\mathbb{N}$ whenever $\gamma(h) \rightarrow 0$ as $h \rightarrow \infty$.

There are several methods that automates computation of $\operatorname{var}(\bar{X})$. In physics, the most well known automated method for standard error estimation is perhaps dependent bootstrapping [18]. Dependent bootstrapping is useful when $n$ is small or if $n$ is large and the required precision is small. The main advantage of bootstrapping methods over the present method is their flexibility. Since bootstrap methods are popular, much is known about their applications, for example, Parr [27] has shown that Frechét differentiability is a sufficient condition that the independent bootstrap works. According to Politis and Romano [18], dependent bootstrapping has asymptotically valid procedures even for multivariate parameter spaces. However, high precision estimates for large data sets are often needed. Flyvbjerg and Petersen [7] proposed an alternative method to automate computation. They proposed an automation by providing a confidence interval to test for normality of $\mathbf{X}_{k}$ using $\widehat{\sigma}^{2}$. Typically, this method works well since $\gamma(h) \propto$ $1 / h$ is commonly used in physics. However, this is not always

\footnotetext{
${ }^{1}$ That constant is $\sigma_{0}^{2}$, as can be proved by iteratively using Proposition 1 .
} 
the case, and for automations operating without supervision, it is possible to provide improvements. For example, stability of the method depends on the shape of the covariance $\gamma(h)$. This can fail for certain types of correlation structures, for example, oscillatory AR(2)-like processes introduced here. The automation works for causal $\operatorname{AR}(p)$ processes for any $p$, and places no assumption on the shape of $\gamma(h)$. In addition, this paper provides updates that make it convenient to use the method for multithread computing. Another alternative method is the Gamma method proposed by Wolff [28]. The Gamma method works well with correct setup, with errors claimed to be lower than those of the automatic blocking method proposed here. Wolff [28] claims that the Gamma method works for other types of correlation structures than exponential decaying types. But, it may be necessary to set up the method's integration window manually. Wolff provides suitable tools for the purpose, and explains that it seems impossible to design automatic windowing that is adequate in all possible cases. As such, it is possible to introduce a fully automated method. In contrast to Wolff [28] recommendation, Lee et al. [29] are proponents of a method that Wolff has called binning (which is essentially a blocking method). Lee et al. proposed inequalities that can be used to automate calculations. However, this approach requires estimates that may or may not be available. The automated blocking method has none of the complications mentioned above.

The proposed automation uses exactly two approximations; namely, that (1) $\Sigma_{j}$ is diagonal and (2) calculated to leading order in $1 / n_{k}$. In practice, approximation (2) can be avoided because an exact expression for the covariance matrix is provided by Proposition 5. However, the method will often fail when approximation (1) is dropped. Consequently, I recommend keeping it as a minimum. The problem is that inversion of $\Sigma_{j}$ can become difficult because the condition number often become very large as $n$ increases. However, using this approximation is not a problem because Proposition 6 guarantees that the expected error of approximation (1) is precisely zero. And, if $n_{k}$ is large, then (2) is not a problem because then $\left(1 / n_{k}\right)^{j} \ll\left(1 / n_{k}\right)^{i}$ for all $i<j$. The bottom line is that $M_{j}$ must be computed using a diagonal covariance matrix $\operatorname{diag}\left(s_{j}, s_{j+1}, \ldots, s_{d-1}\right)$ but it is up to the end user whether he/she sets $s_{i}=\operatorname{var}\left(\widehat{\gamma}_{i}(1)\right)$ using Corollary 3 or expanding to leading in order by setting $s_{i}=\sigma_{i}^{4} / n_{i}$ for all $j \leqslant i \leqslant d-1$. In this paper I opted for $s_{i}=\sigma_{i}^{4} / n_{i}$ because the tests I have performed showed found no effect of higher precision $(p=0.72$, $\mathrm{t}$ test $)$.

Using $\operatorname{AR}(p)$ process for method validation is natural in this context because it is possible to quantify both the error due to (i) the method and (ii) due to the sampling of the data. It would have been impossible to encompass the error due to sampling if the estimates had been compared to high precision estimates from another industry standard method. The error estimates are empirical rather than analytical, but one drawback is that it is only possible to validate the method on a finite number of problems. AR(1) and AR(2) processes were chosen because Wold decomposition says that the random component of any time series can be expressed as an autoregressive model [6]. AR(1) and AR(2) correlation functions are the two most common ones encountered in modeling of time series. The two textbook cases, quantum dot and the Ising model, show that their correlation structures were similar to the AR(1) processes. Using Eq. (42), the results show that the accuracy is as follows: With almost no data available, end users can expect that the estimates are of correct order of magnitude (since $\epsilon^{2}=e^{\beta_{0}}$ if $n=\tau$ ). The expected accuracy increases to produce the first correct digit already at circa $n=10^{4}$ and $10^{5}$ observations for the Ising model and quantum dot, respectively. While it is expected that the second digit is also correct if $n$ circa $10^{6}$ and $10^{8}$ for the Ising model and quantum dot, respectively. This means that the convergence of the relative error to zero is slower than the claimed value for the Gamma method [28]. However, unlike the estimates due to Wolff [28], Table II gives regression results, thus providing a measure on all sources of error (even the errors made by the end users in sampling of the data). In practice, the physics application shows that the estimates are similar to those of dependent bootstrapping and the Flyvbjerg and Petersen [7] blocking method, regardless of $n$ (see Fig. 4), is fully automatic and works in $O(n)$ time.

\section{CONCLUSION AND PERSPECTIVES}

A rigorous proof of the blocking method (Flyvbjerg and Petersen [7]) is a main result of this study. That method has become one of the industry standards for estimating standard errors $\operatorname{var}(\bar{X})^{1 / 2}$ of the mean whenever the number of observations is large. Second, the proof gives an automated implementation that eliminates the need for human intervention. The method uses Fisherian inference to propose a hypothesis test that can be used to determine the estimate of the standard error. The method has complexity $O(n)$, and works for all common covariance structures in natural sciences. This should first and foremost appeal to researchers in computational physics, but also in other sciences, since the study conforms to the standard rigor of modern mathematics and introduces terminology standard in the other sciences. By being automated and complexity $O(n)$, the present method is less expensive than other methods for standard error estimation of the mean used in computational physics (source code is available from [8]).

The paper proposes prospects for more research. Proposition 1 shows that the behavior of any blocking method is determined by the set $\left\{\gamma_{k}(1)\right\}_{k=0}^{d-1}$. However, more advanced estimation is needed to use the result for efficient estimation of $\operatorname{var}(\bar{X})$. The problem is that for large $k$, the data available to estimate $\gamma_{k}(1)$ are small and, consequently, any classical Fisherian estimation is inappropriate. Accordingly, shrinkage estimation or Bayesian estimation may be used. The result is interesting for applications because the truncation error of blocking methods can be expressed in terms of $\left\{\gamma_{k}(1)\right\}$. Therefore, professional error bounds may be provided by developing the mathematics further. Or, better yet, it may be possible to estimate the errors, which would provide significant benefits to end users. Furthermore, it is probably possible to relax the requirements of Theorem 2 because work is constantly being done on central limit theorems. Finally, it would be useful to classify all the Markov chain Monte Carlo methods that are common in computational physics (see for 
example [30]), such that it is more clear for which methods Theorem 2 continues to hold.

\section{ACKNOWLEDGMENTS}

The author is indebted to Professor M. Hjorth-Jensen (Department of Physics, University of Oslo) and Associate Professor H. Flyvbjerg (Department of Micro- and Nanotechnology, Technical University of Denmark) for valuable support during the development of the results. Thanks go to Professor $\varnothing$. Borgan and Professor A. Rygh Swensen (Department of Mathematics, University of Oslo) for helpful comments to drafts of the manuscript. Professor G. L. Jones (School of Statistics, University of Minnesota) and Professor R. C. Bradley (Department of Mathematics, Indiana University) had technical comments that were helpful and the author is grateful to K. Ravn (University of Oslo) for contributing essential pattern-finding skills for Proposition 5. Finally, I am grateful to two anonymous reviewers for their helpful comments and suggestions.

\section{APPENDIX}

Lemma 6. The sequence $\left\{f_{k}\right\}$ satisfies the following properties:

(1) $f_{k}(i) \leqslant i$ for all $1 \leqslant i \leqslant 2^{k+1}-1$.

(2) $\sum_{i=1}^{2^{k+1}-1} f_{k}(i)=2^{2 k}$.

(3) $f_{k+1}(i)=f_{k}(i)+2 f_{k}\left(i-2^{k}\right)+f_{k}\left(i-2^{k+1}\right)$.
Proof. The first property is obvious. For the second property, use the arithmetic series formula. Write

$$
\begin{aligned}
\sum_{i=1}^{2^{k+1}-1} f_{k}(i) & =1+2+\cdots+2^{k}+\left(2^{k}-1\right)+\cdots+2+1 \\
& =2^{k}+2 \sum_{i=1}^{2^{k}-1} i=2^{k}+2 \frac{2^{k}-1}{2} 2^{k}=2^{2 k} .
\end{aligned}
$$

For the third property, use induction. The base case is satisfied for $k=0$, then,

$$
\begin{aligned}
& 1=f_{1}(1)=f_{0}(1)+2 f_{0}(1-1)+f_{0}(1-2)=1+0+0, \\
& 2=f_{1}(2)=f_{0}(2)+2 f_{1}(2-1)+f_{0}(2-2)=0+2+0, \\
& 1=f_{1}(3)=f_{0}(3)+2 f_{2}(3-1)+f_{0}(3-2)=0+0+1 .
\end{aligned}
$$

For the induction step, suppose hypothesis is true for $k$. If $0 \leqslant$ $i \leqslant 2^{k+1}$, then $f_{k+1}=i$. Moreover, either $0 \leqslant i \leqslant 2^{k}$ or $2^{k} \leqslant$ $i \leqslant 2^{k+1}$. If the former is true, then

$$
f_{k}(i)+2 f_{k}\left(i-2^{k}\right)+f_{k}\left(i-2^{k+1}\right)=i+2 \times 0+0=i .
$$

If the latter is true, then

$$
\begin{aligned}
f_{k}(i) & +2 f_{k}\left(i-2^{k}\right)+f_{k}\left(i-2^{k+1}\right) \\
= & 2^{k+1}-i+2\left(i-2^{k}\right)+0=i .
\end{aligned}
$$

The other cases are proved similarly.
[1] K. F. Riley and M. P. Hobson, Essential Mathematical Methods for the Physical Sciences (Cambridge University press, Cambridge, 2011).

[2] The Editors, Phys. Rev. A 83, 040001 (2011).

[3] J. L. Devore and K. L. Berk, Modern Mathematical Statistics with Applications, 2nd ed. (Springer, London, 2012).

[4] J. N. McDonald and N. A. Weiss, A Course in Real Analysis, 2nd ed. (Academic, Oxford, 2013).

[5] M. H. DeGroot and M. J. Schervish, Probability and Statistics, 4th ed. (Pearson Education, Harlow, 2014).

[6] R. H. Shumway and D. S. Stoffer, Time Series Analysis and Its Applications with $R$ Examples, 4th ed. (Springer, Cham, 2017).

[7] H. Flyvbjerg and H. Petersen, J. Chem. Phys. 91, 461 (1989).

[8] Python, R, and $\mathrm{C}++$ code is available: www.github.com/ computative/block.

[9] P. J. Brockwell and R. A. Davis, Introduction to Time Series and Forecasting, 3rd ed. (Springer, Basel, 2016).

[10] A. Agresti, Foundations of Linear and Generalized Linear Models, 1st ed. (Wiley, Hoboken NJ, 2015).

[11] A. Gelman, J. B. Carlin, H. S. Stern, D. B. Dunson, A. Vehtari, and D. B. Rubin, Bayesian Data Analysis, 3rd ed. (CRC Press, Boca Raton, FL, 2014).

[12] I. A. Ibragimov, Teor. Veroyatnost. i Primenen. 20, 135 (1975).

[13] R. C. Bradley, Rocky Mountain J. Math. 17, 95 (1987).

[14] B. Efron, Am. Stat. 40, 1 (1986).

[15] A. Mathai and S. B. Provost, Quadratic Forms in Random Variables (Marcel Dekker, New York, 1992).
[16] D. C. Lay, Linear Algebra and Its Applications, 4th ed. (Addison-Wesley, Boston, 2012).

[17] M. Hazewinkel, Encyclopedia of Mathematics (Kluwer, Dordrecht, 1993).

[18] D. N. Politis and J. P. Romano, J. Am. Stat. Assoc. 89, 1303 (1994).

[19] C. W. Gardiner, Handbook of Stochastic Methods for Physics, Chemistry and the Natural Sciences, 2nd ed. (Springer, Berlin, 1985).

[20] M. Plischke and B. Bergersen, Equilibrium Statistical Physics, 3rd ed. (World Scientific, New Jersey, 2006).

[21] N. Metropolis, A. W. Rosenbluth, M. N. Rosenbluth, A. H. Teller, and E. Teller, J. Chem. Phys. 21, 1087 (1953).

[22] D. N. Politis and H. White, Econom. Rev. 23, 53 (2006).

[23] M. Hazewinkel, Encyclopedia of Mathematics (Kluwer, Dordrecht, 1989).

[24] E. J. Borowski and J. M. Borwein, Dictionary of Mathematics (Collins, London, 1989).

[25] C. Stein, Proceedings of the Third Berkeley Symposium on Mathematical Statistics and Probability, Contributions to the Theory of Statistics, Vol. 1 (Univ. of Calif. Press, 1956), pp. 197-206.

[26] J. Schäfer and K. Strimmer, Stat. Appl. Genet. Mol. Biol. 4, 1 (2005).

[27] W. C. Parr, Stat. Probab. Lett. 3, 97 (1985).

[28] U. Wolff, Comput. Phys. Commun. 156, 143 (2004).

[29] R. M. Lee, G. J. Conduit, N. Nemec, P. López Ríos, and N. D. Drummond, Phys. Rev. E. 83, 066706 (2011).

[30] G. L. Jones, Probab. Surveys 1, 299 (2004). 